\title{
Title: Motor Protein MYO1C is Critical for Photoreceptor Opsin Trafficking and Visual Function
}

Authors: Ashish K. Solanki ${ }^{1 \#}$, Stephen Walterhouse ${ }^{1 \#}$, René Martin², Elisabeth Obert ${ }^{3}$, Ehtesham Arif', Bushra Rahman ${ }^{1}$, Barbel Rohrer ${ }^{3,4}$, Joshua Lipschutz ${ }^{1,4}$, Rupak D. Mukherjee $^{5}$, Russell A. Norris ${ }^{6}$, Jeffery Sundstrom ${ }^{7}$, Hans-Joachim Knölker², Shahid Husain $^{3 *}$, Manas R. Biswal ${ }^{8 *}$, Deepak Nihalani ${ }^{9 *}$ and Glenn P. Lobo ${ }^{1,3 *}$

Affiliations: ${ }^{1}$ Department of Medicine, Medical University of South Carolina, Charleston, SC 29425, USA. 'Faculty of Chemistry, Technische Universität Dresden, Bergstraße 66, 01069 Dresden, Germany. ${ }^{3}$ Department of Ophthalmology, Medical University of South Carolina, Charleston, SC 29425, USA. ${ }^{4}$ Ralph H. Johnson VA Medical Center, Division of Research, Charleston, SC 29420, USA. ${ }^{5}$ Department of Surgery, College of Medicine, Medical University of South Carolina, Charleston, SC 29425, USA. ${ }^{6}$ Department of Regenerative Medicine and Cell Biology, Children's Research Institute, Medical University of South Carolina, Charleston, SC 29425, USA. ${ }^{7}$ Penn State Eye Center, 200 Campus Dr., Suite 800, Hershey, PA 17033. USA. ${ }^{8}$ Department of Pharmaceutical Sciences, Taneja College of Pharmacy, University of South Florida, Tampa, FL 33612, USA. ${ }^{9}$ National Institute of Diabetes and Digestive and Kidney Diseases (NIDDK), National Institutes of Health, Bldg 2DEM, Room 6085, 6707 Democracy Blvd., Bethesda, MD 20817, USA.

\# Equal contribution

\section{${ }^{*}$ Corresponding Authors}

${ }^{\star}$ Glenn P. Lobo, Ph.D.

Assistant Professor

Department of Medicine

30 Medical University of South Carolina

3170 President Road

32 Drug Discovery Building DDB513

33 Charleston, SC 29425, USA.

34 Office: 843-876-2371

35 Fax: 843-792-8399

36 E-mail: lobo@musc.edu

${ }^{*}$ Deepak Nihalani, Ph.D.

39 Program Director, $\mathrm{KUH}$

$40 \quad$ Bldg 2DEM, Room 6085

416707 Democracy Blvd

42 National Institute of Diabetes and Digestive and Kidney Diseases (NIDDK)

43 National Institutes of Health

44 BETHESDA, MD 20817, USA.

45 Office: 301-555-5555

46 E-mail: nihalanideepak@hotmail.com 
47 *Manas R. Biswal, Ph.D.

48 Assistant Professor

49 Department of Pharmaceutical Sciences

50 Taneja College of Pharmacy, University of South Florida

5112901 Bruce B. Downs Blvd, MDC-30

52 Tampa, FL 33612, USA.

53 Office: 813-974-8333

54 E-mail: biswal@usf.edu

55

56

*Shahid Husain, Ph.D.

Professor

58 College of Medicine

59 Department of Ophthalmology

60 Medical University of South Carolina

61 Charleston, SC 29425, USA.

62 Office: 843-792-2792

63 E-mail: husain@musc.edu

64

65

66 Keywords: Motor protein, Myosin, MYO1C, Rhodopsin, Photoreceptor, Outer Segments,

67 Trafficking, Visual function.

68 


\section{Abstract}

70 Unconventional myosins linked to deafness are also proposed to play a role in retinal cell

71 physiology. However, their direct role in photoreceptor function remains unclear. We

72 demonstrate that systemic loss of the unconventional myosin MYO1C in mice specifically

73 affected opsin trafficking, leading to loss of visual function. Electroretinogram analysis of

74 Myo1c knockout (Myo1c-KO) mice showed a progressive loss of photoreceptor function.

75 Immunohistochemistry and binding assays demonstrated MYO1C localization to

76 photoreceptor inner and outer segments (OS) and identified a direct interaction of

77 rhodopsin with the MYO1C cargo domain. In Myo1c-KO retinas, rhodopsin mislocalized

78 to rod inner segments (IS) and cell bodies, while cone opsins in OS showed punctate

79 staining. In aged mice, the histological and ultrastructural examination of the phenotype

80 of Myo1c-KO retinas showed progressively shorter photoreceptor OS. These results

81 demonstrate that MYO1C is critical for opsin trafficking to the photoreceptor OS and for

82 normal visual function.

83

84

85

86

87

88

89 


\section{Introduction}

91 Protein trafficking within the photoreceptors must occur efficiently and at high fidelity for

92 photoreception, photoreceptor structural maintenance, and overall retinal cell

93 homeostasis. Additionally, it is well-known that proper opsin trafficking is tightly coupled

94 to photoreceptor cell survival and function [1-9]. However, the cellular events that

95 participate in retinal injuries due to improper signaling and protein trafficking to the

96 photoreceptor outer segments (OS), are not yet fully understood. While many proteins

97 are known to play an essential role in retinal cell development and function, the

98 involvement of motor proteins in eye biology is less understood. Identification of genetic

99 mutations in the Myo7a gene associated with retinal degeneration in Usher syndrome

100 suggests that unconventional myosins play a critical role in retinal pigmented epithelium

101 (RPE) and photoreceptor cell function [10, 11]. Unconventional myosins are motor

102 proteins that are proposed to transport membranous organelles along the actin filaments

103 in an adenosine triphosphate (ATP)-dependent manner, and additional roles are currently

104 being discovered [12-14]. The loss of Myo7a primarily affects RPE and OS phagocytosis

105 leading to retinal cell degeneration. However, it is believed that other yet unidentified class

106 I myosins may participate more directly in photoreceptor cell function. Here we present

107 compelling evidence for another unconventional actin-binding motor protein, MYO1C,

108 with its primary localization to photoreceptors that plays an important role in retinal cell

109 structure and function via opsin trafficking to the photoreceptor OS.

110 Rhodopsin and cone pigments in photoreceptor OS mediate scotopic and photopic

111 vision, respectively. The visual pigment rhodopsin is a prototypical G-protein coupled

112 receptor (GPCR) expressed by retinal rods for photon absorption. Light sensitivity is 
113 conferred by 11-cis retinaldehyde, a chromophore that is covalently linked to the K296

114 residue of the opsin protein [15-19] Photon absorption causes a cis to trans

115 conformational shift in the retinaldehyde, leading to structural changes in the opsin protein

116 moiety [6]. This initiates a GPCR signaling pathway/phototransduction cascade, signaling

117 the presence of light. Each photoreceptor cell contains an OS housing the

118 phototransduction machinery, an inner segment (IS) where proteins are biosynthesized,

119 and a synaptic terminal for signal transmission. One of the fundamental steps in vision is

120 the proper assembly of signal-transducing membranes, including the transport and

121 sorting of protein components. A major cause of neurodegenerative and other inherited

122 retinal disorders is the improper localization of proteins. Mislocalization of the dim-light

123 photoreceptor protein rhodopsin is a phenotype observed in many forms of blinding

124 diseases, including retinitis pigmentosa $(\mathrm{RP})$. The proteins that participate in

125 phototransduction (including rhodopsin, transducin, phosphodiesterase [PDE6], or the 126 cyclic nucleotide-gated channels [CNG]) are synthesized in the IS and must be

127 transported through the connecting cilium to the OS. These proteins are either

128 transmembrane or peripherally associated membrane, which are attached to the 129 membrane surface [1-9]. How the transmembrane proteins (e.g., rhodopsin and CNG)

130 and peripherally associated proteins (e.g., transducin and PDE6) traffic through the IS to 131 incorporate eventually in the nascent disc membrane or the photoreceptor outer 132 membrane is not fully understood and constitutes an area of intense research, as 133 improper trafficking of these protein causes retinal cell degeneration and can lead to 134 blindness [1-9]. 

with retinal degeneration. Some of the essential genes involved in either or both of these

137 functions belong to a family of unconventional motor proteins and include MYO3A [20], 138 MYO7A, MYO6, MYO15 [20-22], and MYO5. Recently, it was reported that another 139 unconventional myosin, MYO1C, where mutations affected its nucleotide-binding pocket 140 and calcium binding ability and these were associated with deafness [23]. Importantly, 141 MYO1C was identified in proteomic analysis of the retina and vitreous fluid as part of a 142 protein hub involved in oxidative stress [23-25]. MYO1C is an actin-binding motor protein 143 that is widely expressed in multiple cell types. It participates in a variety of cellular 144 functions, including protein trafficking and translocation [12, 26-28]. As MYO1C has low 145 tissue specificity based on mRNA and protein expression, it remains unclear which cell 146 type is most dependent on MYO1C trafficking function and is affected by the loss of 147 MYO1C.

148 In this study, we systematically analyzed the function of the unconventional motor

149 protein MYO1C in protein trafficking in photoreceptors. We found that a global genetic 150 deletion of Myo1c resulted in a retinal phenotype only, which manifested as a progressive 151 mistrafficking of opsins to the OS. Using retinal lysate from wild-type (WT) mice in co152 immunoprecipitation assays, we showed that MYO1C and rhodopsin directly interact, 153 indicating that opsin was a cargo for MYO1C. Loss of MYO1C promoted a progressive 154 shortening of OSs that was concomitant with a reduction in photoreceptor function, 155 suggesting that MYO1C is critical for maintenance of photoreceptor cell structure and for 156 visual function. Our findings have significant clinical implications for degenerative rod and 157 cone diseases, as mutations in MYO1C or its interacting partners are predicted to affect 
158 retinal health and visual function by altering opsin trafficking to the photoreceptor OS, a

159 fundamental step for maintaining visual function in humans.

\section{Results}

Construction and Validation of Myo1c Null Mice: We previously generated Myo1c

163 floxed mice using the standard knockout strategy [29] (Fig. S1a). Systemic deletion of 164 Myo1c was achieved by crossing Myo1c floxed (Myo1cfl/fl) mice with Actin Cre+ 165 (ActCre+; JAX labs) mice to generate Myo1cfl/fl-ActCre+/- knockout mice (referred to as 166 Myo1c-KO mice in this manuscript). Western blotting of protein lysates from various

167 tissues including kidney, heart, and liver of Myo1c-KO mice showed complete loss of 168 MYO1C, thus confirming the systemic deletion of Myo1c (Fig. S1b). Additionally, 169 immunofluorescence expression analysis of these tissues further confirmed loss of 170 MYO1C protein in Myo1c-KO mice (Figs. S2a-c).

172 Genetic Deletion of Myo1c induced Visual Impairment in Mice: Immunofluorescence

173 analysis showed that MYO1C was enriched in the rod photoreceptor outer (OS) and inner

174 segments (IS) (Fig. 1a), and also in cone photoreceptor OS of wild-type (WT) mice (Fig.

175 1b), but absent in photoreceptors of Myo1c-KO animals (Figs. 1a and 1c). Western blot analysis further confirmed that MYO1C protein was absent in the retinas of Myo1c-KO

177 mice (Fig. 1d). Since mutations or deletion of the motor protein MYO7A were associated 178 with retinal degeneration in Usher syndrome and its animal model, it prompted us to 179 investigate the effect of Myo1c in retinal function. Using electroretinograms (ERGs) [30, 180 31], we tested photoreceptor cell function of Myo1c-KO and WT mice ( $n=8$ mice per 
181 genotype and age-group; 50:50 ratio of male and female) under dark-adapted scotopic

182 conditions. In contrast to WT animals, we observed reduced ERGs for Myo1c-KO mice

183 at different ages. Two month old Myo1c-KO mice showed a significant reduction in the a-

184 wave amplitudes, but not in $\boldsymbol{b}$-wave amplitudes $(p<0.0068$ and $p<0.098$, respectively)

185 (Figs. 2a and 2c). Strikingly, ERG analysis of adult six months old Myo1c-KO mice

186 showed severe loss of retinal function, in which a significant reduction in both $\boldsymbol{a}$ - and $\boldsymbol{b}$ -

187 waves was observed (38-45\% lower than WT animals $\left({ }^{* \star} p<0.005\right.$; Figs. 2 b and $\mathbf{2 d}$ ).

189 Trafficking of Rod and Cone Visual Pigments in Myo1c-KO Mice: Since the

190 phototransduction protein rhodopsin constitutes $85-90 \%$ of photoreceptor OS protein

191 content [32], and as the ERG responses were impaired in Myo1c-KO mice, we

192 hypothesized that the loss of MYO1C might have affected opsin trafficking to the

193 photoreceptor OS. To test this hypothesis, we analyzed retinal sections from WT and

194 Myo1c-KO mice (at 2 and 6 months of age; 5-7 retinal sections per eye from $n=8$ mice

195 per genotype and age-group; 50:50 ratio of male and female), probing for rhodopsin, two

196 types of cone opsins, medium wavelength R/G opsin (M-opsin) and short wavelength S-

197 opsin, rod-specific phosphodiesterase 6b (Pde6b), rod-specific CNGA1, rod arrestin

198 (ARR1), rod transducin (G-protein), and the general cone marker, PNA lectin. In WT mice

199 at 2 and 6 months of age, rhodopsin localized exclusively to the rod OS (Fig. 3a). While

200 majority of rhodopsin trafficked to the OS in two month old Myo1c-KO mouse retinas,

201 some mislocalization to the base of the rod IS and the cell bodies in the outer nuclear

202 layer (ONL) was noted (Fig. 3a; white arrows; rhodopsin levels within individual retinal

203 layers were quantified and shown in Figs. S3a-c). This suggested incomplete opsin 
204 transport/trafficking to photoreceptor OS in the absence of MYO1C. An even more severe

205 mislocalization of rhodopsin to the rod IS and within the ONL was observed in the 6-month

206 old Myo1c-KO mice, suggesting a progressive retinal phenotype in the absence of

207 MYO1C (Fig. 3a; rhodopsin expression within individual retinal layers were quantified and

208 shown in Figs. S3d-e). Staining for the two cone opsins showed that the cone OS were

209 shorter and mis-shaped by two months and this abnormality increased by six months of

210 age (Figs. 3b and 3c). Retinas stained for PNA lectin, showed progressively shorter and

211 mis-shaped cone OS, indicating that cone OS structure was compromised in the absence

212 of MYO1C as these mice aged (Fig. 3d). Cone visual arrestin in WT mice retina typically

213 outlines the entire cell, OS, IS, cell body, axon, and cone pedicle. Staining for cone

214 arrestin in Myo1c-KO animals (2 month of age) confirmed the short and mis-shaped

215 appearance of the cone OS compared to WT retinas at similar ages (Fig. 4a, white

216 arrows). In contrast, staining for Pde6b, a lipidated rod specific protein that trafficks to the

217 OS independently of rhodopsin [33], showed normal trafficking and localization to the rod

218 OS in both WT and Myo1c-KO retinas, at 2 month of age (Fig. $\mathbf{4 b}$ ).

219 The CNG channels are also important mediators in the photoreceptor transduction

220 pathways, and they require proper localization to the OS for normal photoreceptor cell

221 function [5]. Additionally, the absence of CNGA1 or CNGB1 in mice led to decreased ERG

222 responses and progressive rod and cone photoreceptor cell death [5]. Therefore, to rule

223 out alternate mechanisms for the observed functional phenotypes in Myo1c-KO retinas,

224 the retinas of WT and Myo1c-KO mice (3-4 months of age; 5-7 retinal sections per eye

225 from $n=8$ mice per genotype; 50:50 ratio of male and female) were stained with the

226 CNGA1 antibody. This analysis showed that even in the absence of MYO1C, both young 
227 and adult mice retinas showed no defects in the trafficking of CNGA1 protein to OS (Fig.

228 4c; CNGA1 protein distribution in photoreceptor layer quantified and shown in Fig. 4f).

229 The soluble proteins arrestin and transducin exhibit light-dependent trafficking,

230 where in response to light, arrestin migrates to rod OS and transducin translocates to rod

231 IS [34]. To test whether the loss of MYO1C affected rod arrestin (ARR1) and rod G-protein

232 (transducin) localization, we performed IHC staining for these proteins in retinas of light

233 adapted WT and Myo1c-KO mice (3-4 months of age; 5-7 retinal sections per eye from

$234 n=8$ mice per genotype; 50:50 ratio of male and female). These analyses showed that in

235 the presence of light, genetic loss of MYO1C had no negative effect on the trafficking of

236 rod arrestin to the OS and G-protein to the IS and cell bodies in retinas of Myo1c-KO mice

237 (Figs. 4d and 4e; rod ARR1 and transducin protein distribution in photoreceptor layer

238 quantified and shown in Fig. 4f). Using total protein lysates from retinas of WT and

239 Myo1c-KO mice (3-4 months of age; four pooled retinas from $n=2$ mice per genotype) we

240 analyzed protein expression of key retinal proteins in specific retinal cells: CRABLP1

241 (expressed in Müller cells), GNAT1 (expressed in photoreceptors), and PKC $\alpha$ (expressed

242 in retinal bipolar cells). These analyses showed no significant differences in the

243 expression of these genes in the inner or outer-retinal layers of Myo1c-KO mice when

244 compared to WT mice, at 3-4 months of age (Fig. 4g). Although MYO1C could not be

245 detected by immunohistochemical analysis in mouse RPE, functional MYO1C and Myo1C

246 mRNA were reported in human RPE cells [35] and mouse RPE [36], respectively. Since

247 elimination of the motor protein Myo7a in mouse leads to alterations in protein localization

248 in the RPE (RPE65) [37], we stained retinas of young and adult WT and Myo1c-KO mice

249 (5-7 retinal sections per eye from $n=8$ mice per genotype) with an anti-STRA6 antibody, 
250 another RPE-specific protein. This analysis showed that STRA6 expression and

251 localization in the RPE was not affected in the absence of MYO1C (Fig. S4). Since

252 MYO1C is known primarily as a motor protein with a protein trafficking function [14, 23],

253 we next tested the hypothesis that its absence in photoreceptors of Myo1c-KO animals

254 may contribute specifically to the loss of opsin trafficking to the photoreceptor OS.

256 Native Cre+ mice showed no retinal phenotypes: To rule out any Cre+-mediated

257 effects on retinal phenotypes observed in the Myo1c-KO;Cre+ animals, the eyes from 258 native Cre+ mice (3-4 months old; $n=3$ animals) were harvested and subjected to similar

259 histological and immunofluorescence analysis. As compared to age-matched WT mice 260 retinas ( $n=3$ animals), the retinas of Cre+ mice showed no retinal pathology or 261 mislocalization of opsins (Figs. S5a vs. S5b). These analyses support the view that 262 genetic loss of MYO1C affects key components of phototransduction specifically, and this 263 is further manifested in defects in visual function.

265 Myo1c-KO Mice demonstrated Photoreceptor OS Loss: To evaluate further if opsin

266 mistrafficking is associated with structural changes to the retina, histological and

267 transmission electron microscopy (TEM) analyses of retinal sections of young and adult 268 WT and Myo1c-KO mice were performed. In histological sections of retinas (5-7 retinal 269 sections per eye from $n=8$ mice per genotype and age), progressive shortening of rod 270 photoreceptor OS was observed. The OS of adult Myo1c-KO mice at 6 months of age

271 were shorter than the OS of Myo1c-KO mice at 2 months of age, which in turn were 272 shorter than those in WT mice at similar ages (Figs. 5a and 5b; OS lengths quantified 
273 from H\&E sections and represented using spider-plots in Figs. 5c and 5 d; $\left.{ }^{* *} p<0.05\right)$. In

274 comparison to WT mice, the photoreceptors in Myo1c-KO mice were less organized,

275 especially in the 6-month old mice (Fig. 5b), suggesting that loss of MYO1C may 276 progressively affect photoreceptor homeostasis. The retina outer nuclear layer (ONL)

277 thickness between genotypes at both ages revealed no significant reduction in nuclear 278 layers in Myo1c-KO animals compared to WT mice (ONL thickness quantified from H\&E 279 stained sections and represented using spider-plots in Figs. 5e and 5f).

281 Ultrastructural TEM analysis showed shorter photoreceptor OS in Myo1c-KO mice:

282 To evaluate the structure of rod photoreceptors, ultrastructural analysis using TEM was

283 performed ( $n=6$ retinal sections per eye from $n=8$ mice per genotype and age). While the 284 rod photoreceptor OS in the WT mice showed normal elongated morphology, they 285 appeared slightly shorter in Myo1c-KO mice at two months of age ( ${ }^{*} p<0.05$; Fig. 6a; rod 286 OS lengths quantified in Fig. 6e). Specifically, comparing Myo1c-KO with WT mouse rod 287 OS lengths at six months of age demonstrated that OS segment lengths in Myo1c retinas 288 were significantly $(36-45 \%)$ shorter than those of WT mice $\left({ }^{* *} p<0.005\right.$; Fig. $6 \mathbf{b}$; rod OS 289 lengths quantified in Fig. 6e). Ultrastructurally, the cone OS in the Myo1c-KO mouse 290 retina were shorter and had lost their typical cone shape (Fig. 6c vs. 6d; cone OS lengths 291 quantified in Fig. 6f), confirming the mis-shaped cone OS phenotype identified by 292 immunohistochemistry (Figs. 3b-d). These results suggest that the lack of MYO1C 293 resulted in progressively severe opsin mislocalization (Figs. 3a-d), and shorter 294 photoreceptor OS (Figs. 5 and 6), thus supporting the observed decrease in visual 295 function by ERG (Fig. 2). 
Molecular inhibition of MY01C motor function by PCIP in mice affected Opsin

297 trafficking: To confirm a direct role for the motor protein MYO1C in opsin trafficking and

298 that the loss of MYO1C contributed specifically to opsin mistrafficking in photoreceptors,

299 we molecularly inhibited MYO1C in vivo using PCIP (pentachloropseudilin) which

300 specifically can inhibit the motor activity of MYO1C [38-41]. To achieve this, a single dose

301 of PCIP in DMSO $(5 \mathrm{mg} / \mathrm{kg})$ was injected retro-orbitally into the right eye of WT animals

$302(n=2)$. A control set of WT animals $(n=2)$ received vehicle control/diluent (DMSO) under

303 similar conditions. Post 7-8 hours injection, mice were euthanized and both eyes were

304 harvested and fixed in PBS buffered 4\% paraformaldehyde. Retinal cell phenotype and

305 opsin trafficking in PCIP and DMSO injected animals were assessed using retinal

306 histology and immunofluorescence to assess rod and cone opsin trafficking to the OS. In

307 photoreceptors of mice eyes injected with the vehicle control DMSO, rhodopsin localized

308 exclusively to the rod OS (Fig. 7a). In contrast, the mice injected with PCIP showed

309 mislocalization of rhodopsin to the base of the rod IS and the cell bodies in the ONL,

310 demonstrating incomplete opsin trafficking to the photoreceptor OS in PCIP injected mice

311 retinas (Fig. 7a; white arrows). Observation of retinal phenotypes in the left eyes of these

312 animals indicated that injected PCIP was systemically distributed (Fig. 7a). Staining for

313 the R/G cone opsins (M-opsins) in retinas of these animals showed that in comparison to

314 the control mice the cone OS of PCIP injected mice were shorter and lost their typical

315 elongated cone morphology (Fig. 7b). The histological analysis of retinal sections showed

316 significant shortening of photoreceptor OS in mice retinas injected with PCIP (Fig. 7c).

317 Additionally, the quantification of retinal ONL thickness in PCIP injected mice showed that

318 ONL was slightly thinner in comparison to control mice (ONL thickness quantified from 
319 H\&E sections and represented using spider-plots in Fig. 7d). OS lengths were

320 significantly shorter in PCIP injected mice ( ${ }^{*} \mathrm{p}<0.05$; Fig. 7c; quantified from H\&E sections

321 and represented using spider-plots in Fig. 7e). These retinal phenotypes were similar to

322 those observed in the retinas of Myo1c-KO mice at two and six months (Figs. 3, 5a, and

323 6). Collectively, these results indicate that MYO1C is critical for opsin trafficking to

324 photoreceptor OS and its loss specifically affects opsin trafficking, photoreceptor cell

325 homeostasis, and visual function.

327 MY01C Directly Interacted with Rhodopsin: Since the loss of MYO1C resulted in

328 retinal function defects with significant alterations in the localization of opsins, we next

329 evaluated whether MYO1C exerted this effect through a physical interaction with

330 rhodopsin. Immunoprecipitation analysis using WT and Myo1c-KO mice retinas ( $n=6$

331 retinas pooled from $n=3$ animals per genotype, respectively) demonstrated that rhodopsin

332 was pulled down using MYO1C antibody, and this interaction was confirmed in a

333 reciprocal fashion (Fig. 8a; Co-IP flow-chart schematic shown in Fig. S6). Using a

334 baculovirus-produced purified recombinant mouse MYO1C protein in an overlay assay,

335 we demonstrated that MYO1C directly interacted with rhodopsin, where opsin was

336 immunoprecipitated both from mouse retinal lysate or HEK293 cells transfected with

337 pCDNA3 Rod Opsin in order to overexpress Rhodopsin (schematic representation in Fig.

338 S6). Immunoprecipitated rhodopsin was subjected to western blotting and probed with

339 purified recombinant full-length MYO1C (MYO1C FL; Fig. 8b and schematic in Fig. S6)

340 or GFP-MYO1C-790-1028 (MYO1C tail domain, also known as the cargo domain; Fig.

$3418 \mathrm{c}$ and Fig. S6) [13]. Post-incubation, the interaction of immobilized rhodopsin to Myo1c 
342 was probed using a MYO1C antibody. The immunoblot analysis of the over-layered

343 MYO1C showed significant binding of both MYO1C proteins, full-length and the tail

344 domain, at the rhodopsin band, indicating a direct interaction between the two proteins

345 (Figs. 8b and 8c). Interestingly, the interaction of MYO1C was noted with various

346 multimers of rhodopsin, which further indicated that opsin is a cargo for MYO1C (arrows

347 in Figs. 8b and 8c).

349 Genetic Deletion of Myo1c did not affect Systemic Organs in Mice: Finally, to

350 determine if the global deletion of Myo1c affected other organs, we harvested major

351 systemic organs, including liver, heart, and kidney of 2 month old Myo1c-KO and WT

352 mice ( $n=4$ per genotype), and performed histological analyses. Notably, Myo1c-KO mice

353 developed and reproduced normally with no observable histological differences between

354 the control and Myo1c-KO genotypes (Figs. S7a-c). To further confirm that there were

355 no functional defects in these systemic organs, we performed ECHOcardiogram (heart

356 function), quantified protein/albumin levels in urine (kidney function), and measured

357 Alanine Aminotransferase/ALT enzyme levels (liver function), in Myo1c-KO mice ( $n=4$

358 mice per individual functional analysis) and compared these values to their WT littermates

359 ( $n=4$ mice per individual functional analysis). All of these analyses showed no pathological

360 defects in systemic organs of Myo1c-KO animals when compared to the age-matched

361 WT littermates (Figs. S7a'-c' and S8). Overall, these results indicate that except for the

362 retinal phenotypes, Myo1c-KO animals retained normal physiology of the systemic organs

363 examined. 


\section{Discussion}

366 The trafficking of the G-protein coupled receptor (GPCR) Type II Opsins from the

367 photoreceptor IS to the OS represents a critical event in the initiation of phototransduction

368 for visual function in vertebrates. Our work identified for the first time an unconventional

369 motor protein, MYO1C, as a novel trafficking regulator of both rod and cone opsins to the

370 photoreceptor OS in mice. In this study, based on MYO1C localization within the IS and

371 OS of photoreceptors, and using a whole-body Myo1c-KO mouse model, we functionally

372 identified MYO1C as a novel component of retinal physiology and was specifically found

373 to be involved in photoreceptor cell function. Retinal analysis of Myo1c-KO mice identified

374 opsins as novel cargo for MYO1C. In the absence of MYO1C, both young and adult

375 Myo1c-KO mice showed impaired opsin trafficking, where rhodopsin was retained in the

376 photoreceptor IS and the cell bodies. In contrast, cone opsins showed no retention in the

377 cell body or mistrafficking to other retinal cell layers, although staining patterns revealed

378 deformed cone OS shapes. These two phenotypes manifested as a progressive decline

379 of visual responses in the rod ERGs and shorter photoreceptor OS lengths as Myo1c-KO

380 animals aged, indicating a progressive retinal phenotype. Interestingly, trafficking of other

381 OS proteins (CNGA1, arrestin, and transducin) were largely unaffected in the absence of

382 MYO1C. The genetic deletion of Myo1c only affected retina, and the other systemic

383 organs examined, including heart, liver, and kidney, remained unaffected. Use of PCIP

384 as an allosteric inhibitor of MYO1C ATPase and motor activity resulted in retinal

385 phenotypes similar to those observed in Myo1C-KO mice and thus confirmed that MYO1C

386 plays a critical role in the trafficking of opsin to the photoreceptor OS. Overall, our data

387 points to a novel mechanism by which MYO1C regulates opsin trafficking from the 
388 photoreceptor IS to OS, a critical event for photoreceptor function and long-term

389 photoreceptor cell homeostasis. Our study identifies an unconventional motor protein

390 MYO1C as an essential component of mammalian photoreceptors, where it plays a

391 canonical role in promoting opsin trafficking and maintaining normal visual function.

393 MY01C and Other Opsin Trafficking Proteins: Myo1c-KO mice exhibited rhodopsin

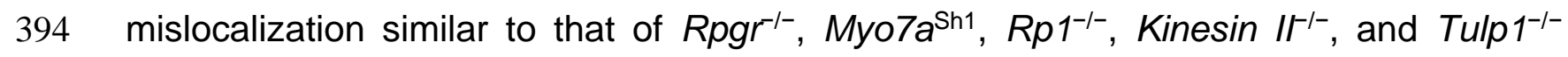
395 mutant mice [1-9]. Since MYO1C primarily localized to photoreceptor IS and OS, is known 396 to be involved in protein trafficking, and uses actin as a track [14, 23], we hypothesized 397 that MYO1C participates in the movement of opsins from IS to the OS of photoreceptors.

398 This hypothesis was supported by the observation that the rod opsins were mislocalized 399 to IS and cell bodies. Defective assembly of cone OS in Myo1c-KO mice suggests that 400 this phenotype is caused by an aberrant protein transport with OS degeneration as a 401 secondary event. The normal ultrastructure of photoreceptors in our Myo1c-KO mice 402 suggests that the retinal abnormalities in these animals were not due to structural defects 403 in photoreceptors per se, but instead were induced by aberrant motor function leading to 404 opsin mislocalization.

MY01C Contributed to Phototransduction and Retinal Homeostasis: The opsin

407 molecules and other phototransduction proteins are synthesized in the cell body of the 408 photoreceptor [42, 43]. They are then transported to the distal IS [44] and subsequently 409 to the OS. Little is known about these transport processes and the molecular components 410 involved in this process [1-9]. The localization of MYO1C in the rod photoreceptors' IS 
411 and OS, and in cone OS, suggested that opsins may utilize this molecular motor for

412 transport to the OS. The immunohistochemical analysis of Myo1c-KO animals indicated

413 that while rod and cone opsins trafficked to the OS, significant mislocalization was noted

414 for rhodopsin in the IS and cell bodies in the ONL (Fig. 2). Since they represent plasma

415 membrane structural proteins, cone opsins presumably contribute to the cone OS stability

416 and rhodopsin to the rod OS formation and stability [7]. Hence, photoreceptor OS

417 shortening/degeneration in Myo1c-KO mice may be attributed, in large part, to the

418 mistrafficking of opsins to the IS or a progressive reduction of opsins in the OS membrane.

419 Notably, the pattern of opsin mislocalization observed in Myo1c-KO mice closely

420 resembled the retinal phenotype observed in our previously reported Tulp1-KO mice [4,

421 45], Cnga3 $^{-1-}$ mice [5], Lrat $^{-1-}$ and Rpe65 $^{-1-}$ mice $[3,8,9]$, GC1-KO mice [1, 6], and, to

422 some extent in $\mathrm{CFH}$ (complement factor $\mathrm{H}$ )-KO animals [2]. Importantly, in all these

423 studies, photoreceptor OS were unstable, and significant degeneration was noted.

424 However, because $85-90 \%$ of OS protein is rhodopsin, the mislocalization of other less

425 abundant proteins cannot be ruled out in the photoreceptors of Myo1c-KO mice.

427 Contributions from Other Motor proteins in Opsin trafficking: Although this study

428 demonstrates mistrafficking of opsins due to a loss of MYO1C, the majority of opsin was

429 still correctly localized, suggesting that contribution or compensation from other myosins

430 cannot be ruled out. Nevertheless, the contributions from MYO1C were highly significant

431 as its genetic deletion showed specific physiological defects in mouse retinas. It is likely

432 that some redundancy exists among molecular motors, and several known candidates

433 might compensate for the lack of MYO1C in photoreceptor function. However, the qPCR 
434 analysis of the retinas from WT and Myo1c-KO mice did not suggest compensation from

435 other family myosin 1 members (Fig. S9). Interestingly, the upregulation of Myo1f in our

436 study was unable to rescue the Myo1c retinal phenotype suggesting that Myo1f is unable

437 to compensate for the functional loss of Myo1c in retina (Fig. S9). However,

438 compensation by other motor proteins, including the members of kinesin superfamily [46,

439 47], myosin VIIa, and conventional myosin (myosin II) [48, 49], which have also been

440 detected in the RPE and retina, cannot be ruled out and need further investigation.

441 Overall, these results support a direct role for MYO1C in opsin trafficking in the

442 photoreceptor cells of the retina and provide evidence that defective protein transport

443 pathways are a pathologic mechanism responsible for OS degeneration and decreased

444 visual function in these mice.

446 Methods

447 Materials: All chemicals, unless stated otherwise, were purchased from Sigma-Aldrich

448 (St. Louis, MO, USA) and were of molecular or cell culture grade quality.

Myo1c-knockout (Myo1c-KO) Mouse Model: Mice were kept with ad libitum access to

451 food and water at $24^{\circ} \mathrm{C}$ in a $12: 12 \mathrm{~h}$ light-dark cycle. All mice experiments were approved

452 by the Institutional Animal Care and Use Committee (IACUC protocol \#00780; G.P.L.) of

453 the Medical University of South Carolina, and performed in compliance with ARVO

454 Statement for the use of Animals in Ophthalmic and Vision Research. We have previously

455 generated Myo1c transgenic mice (Myo1cfl/fl) in C57BL/6N-derived embryonic stem 
456 cells, flanking exons 5 to 13 of the mouse Myo1c gene, which has allowed us to

457 specifically delete all Myo1c isoforms in a cell-specific manner [29]. Here a complete

458 Myo1c-knockout was generated by crossing Myo1cfl/fl mice with an F-actin Cre mouse

459 strain (B6N.FVB-Tmem163Tg(ACTB-cre)2Mrt/CjDswJ) obtained from Jackson Labs. We

460 will refer to the Myo1cfl/fl x f-actin Cre cross as Myo1c knockout (Myo1c-KO) mice. For

461 this study, the Myo1c-KO mice were crossed onto a C57BL/6J background to avoid 462 potential problems with the Rd8 mutation (found in C57BL/6N lines) [50]. Equal numbers 463 of male and female mice (50:50 ratio) were used per group and time-point.

Immunohistochemistry and Fluorescence Imaging: Light-adapted mice were

466 euthanized and eyes immediately enucleated. Eyes were fixed in 4\% paraformaldehyde

467 buffered with $1 \mathrm{X}$ PBS for 2 hours at $4^{\circ} \mathrm{C}$ using established protocols [58]. After fixation,

468 samples were washed in 1X PBS and embedded in paraffin and processed (MUSC

469 Histology core facility). Sections $(10 \mu \mathrm{m})$ were cut and transferred onto frost-free slides.

470 Slide edges were lined with a hydrophobic marker (PAP pen) and deparaffinized using

471 xylene and processed through ethanol washes before blocking for 1-2 hours at RT.

472 Blocking solution (1\% BSA, 5\% normal goat serum, 0.2\% Triton-X-100, 0.1\% Tween-20

473 in 1X PBS) was applied for 2 hours in a humidified chamber. Primary antibodies were

474 diluted in blocking solution as follows: anti-rhodopsin (1:500, Abcam, 1D4), anti-Myo1c

475 (1:100), cone-arrestin (1:250, Millipore-Sigma, St. Louis, MO), conjugated PNA-488

476 (1:2000, Molecular Probes, Eugene, OR), anti-red/green cone opsin (M-opsin; 1:500;

477 Millipore, St. Louis, MO), anti S-opsin (1:500, Millipore/Sigma, St. Louis, MO), ZO1

478 (1:2000, Invitrogen), Pde6b (1:300, ThermoFisher), CNGA1 (1:250, Abcam), rod arrestin 
479 (1:250, Invitrogen), Stra6 (1:250, Millipore-Sigma), CRALBP (1:100, Invitrogen), rod

480 transducin (1:250, Santa Cruz), and 4',6-diamidino-2-phenylendole (DAPI; 1:5000,

481 Invitrogen) or Hoechst (1:10,000, Invitrogen) was used to label nuclei. All secondary

482 antibodies (Alexa 488 or Alexa 594) were used at 1:5000 concentrations (Molecular

483 Probes, Eugene, OR). Optical sections were obtained with a Leica SP8 confocal

484 microscope (Leica, Germany) and processed with the Leica Viewer software. All

485 fluorescently labeled retinal sections on slides were analyzed by the BioQuant NOVA

486 Prime Software (R \& M Biometrics, Nashville) and fluorescence within individual retinal

487 layers quantified using Image $\mathrm{J}$ or Fiji (NIH).

489 Measurement of Photoreceptor ONL thickness and OS lengths: The lengths of the 490 photoreceptor OS in WT and Myo1c-KO animals (from H\&E sections of retinas) were 491 imaged (Keyence BZ-X800 microscope) and measured at 12 consecutive points (at 150 $492 \mu \mathrm{m}$ distances) from the optic nerve (ON). The OS length was measured from the base of 493 the OS to the inner side of the retinal pigment epithelium. The total number of layers of 494 nuclei in the ONL of retinal sections through the optic nerve $(\mathrm{ON})$ was imaged (Keyence 495 BZ-X800 microscope) and measured at 12 locations around the retina, six each in the 496 superior and inferior hemispheres, starting at $150 \mu \mathrm{m}$ from the ON. Retinal sections $(n=$ 497 5-7 retinal sections per eye) from $n=8$ mice for each genotype and time-point were 498 analyzed. Two-way ANOVA with Bonferroni post-tests compared Myo1c-KO to WT mice, 499 at each segment measured. 
502 We and others have previously shown that the natural compound pentachloropseudilin

503 (PCIP) acts as an allosteric inhibitor of MYO1C ATPase and motor activity [38-41, 51-53].

504 To test whether the inhibition of MYO1C function by PCIP affects opsin trafficking, PCIP

505 was retro-orbitaly injected $(5 \mathrm{mg} / \mathrm{kg}$ body weight) into the right eye of two-month old WT

506 animals $(n=2)$. At this concentration, PCIP was observed to inhibit all Myo1c isoforms

507 without affecting non-Myo1 Myosins [35, 38, 40, 41, 51-56]. The control set of WT animals

$508(n=2)$ received retro-orbital injections of vehicle control (DMSO). Post 7-8 hours injection,

509 mice were euthanized, both eyes were harvested, and fixed in 4\% PFA for histological

510 analysis.

512 ERG Analysis: Dark-adapted WT and Myo1c-KO mice (50:50 ratio of male and female;

$513 n=8$ each genotype) at 2 month of age (young mice; early time-point), and 6 month of age

514 (end time-point) were anesthetized by intraperitoneal injection of a ketamine/xylene

515 anesthetic cocktail (100 mg/kg and $20 \mathrm{mg} / \mathrm{kg}$, respectively) and their pupils were dilated

516 with $1 \%$ tropicamide and $2.5 \%$ phenylephrine $\mathrm{HCl}$. ERGs were performed under dim red-

517 light in the ERG rooms in morning (8am-12noon). Scotopic ERGs were recorded with a

518 computerized system (UTASE-3000; LKC Technologies, Inc., Gaithersburg, MD, USA),

519 as previously described [57-59].

521 TEM analysis of retinas: Eyecups at the indicated time-points were harvested and fixed

522 overnight at $4^{\circ} \mathrm{C}$ in a solution containing $2 \%$ paraformaldehyde $/ 2.5 \%$ glutaraldehyde

523 (buffered in $0.1 \mathrm{M}$ cacodylate buffer). Samples were rinsed in the buffer ( $0.1 \mathrm{M}$ cacodylate

524 buffer). Post-fixative $2 \% \mathrm{OsO}_{4} / 0.2 \mathrm{M}$ cacodylate buffer 1 hour at $4^{\circ} \mathrm{C}$, followed by $0.1 \mathrm{M}$ 
525 cacodylate buffer wash. The samples were dehydrated through a graded ethanol series

526 and then embedded in epon (EMbed 812; EM Sciences). For TEM analysis, each eye

527 ( $n=6$ individual eyes from $n=6$ animals of each genotype) was cut in half before

528 embedding in epon blocks. Sections were parallel to the dorsoventral meridian and near

529 the optic nerve $(\mathrm{ON})$. The cured blocks were sectioned at 0.5 microns (semi-thin plastic

530 sections) and stained with $1 \%$ toluidine blue to orient the blocks to the required specific

531 cell types. The blocks were trimmed to the precise size needed for ultrathin sectioning.

532 The blocks were cut at $70 \mathrm{~nm}$ and gathered on 1-micron grids. The grids were air-dried,

533 stained with uranyl acetate for 15 minutes, lead citrate for 5 minutes, and rinsed between

534 each stain. They were allowed to dry and imaged with a JEOL 1010. Images were

535 acquired with a Hamamatsu camera and software. All samples were processed by the

536 Electron Microscopy Resource Laboratory at the Medical University of South Carolina, as

537 previously described [57].

539 Western Blot Analysis and Densitometry: Total protein from cells or mouse tissues

540 ( $n=3$ per genotype) were extracted using the M-PER protein lysis buffer

541 (ThermoScientific, Beverly, MA) containing protease inhibitors (Roche, Indianapolis, IN).

542 Approximately $25 \mu \mathrm{g}$ of total protein was electrophoresed on 4-12\% SDS-PAGE gels and

543 transferred to PVDF membranes. Membranes were probed with primary antibodies

544 against anti-Myo1c (1:250), CRALBP (1:100, Invitrogen), Rod transducin (1:250, Santa

545 Cruz), PKC $\alpha$ (1:500, Novus Biologicals), and $\beta$-Actin or Gapdh $(1: 10,000$, Sigma) in

546 antibody buffer $(0.2 \%$ Triton X-100, 2\% BSA, 1X PBS) [54,55,70]. HRP conjugated

547 secondary antibodies (BioRad, Hercules, CA) were used at 1:10,000 dilution. Protein 
expression was detected using a LI-COR Odyessy system, and relative intensities of each

549 band were quantified (densitometry) using Image $J$ software version 1.49 and normalized

550 to their respective loading controls. Each western blot analysis was repeated thrice.

552 Co-immunoprecipitation (co-IP) Assays: Co-immunoprecipitation of endogenously

553 expressed proteins (MYO1C and rhodopsin) was performed using mouse retinal extracts.

554 Six retinas of each genotype ( $n=3$ animals of WT and Myo1c-KO) were used for extraction

555 of retinal proteins in $250 \mu \mathrm{L}$ of RIPA buffer (phosphate-buffered saline [PBS] containing

$556 \quad 0.1 \%$ sodium dodecyl sulfate [SDS], $1 \%$ Nonidet P-40, $0.5 \%$ sodium deoxycholate, and

$557100 \mathrm{mM}$ potassium iodide) with EDTA-free proteinase inhibitor mixture (Roche Molecular

558 Biochemicals). Lysates were cleared by centrifugation at $10000 \mathrm{rpm}$ for $10 \mathrm{~min}$ at $4^{\circ} \mathrm{C}$.

559 The prepared lysates were further incubated with anti-Myo1c, anti-rhodopsin, and

560 mouse/rabbit IgG overnight at $4^{\circ} \mathrm{C}$ and further with protein $\mathrm{G}$-coupled agarose beads

561 (ROCHE) for $1-2 \mathrm{~h}$. Beads were then collected by centrifugation at 3000 rpm for 5 min at

$5624^{\circ} \mathrm{C}$, extensively washed in $1 \mathrm{X}$ PBS, and resuspended in SDS gel loading buffer. The

563 proteins were separated on a 10\% SDS-PAGE, transferred to a PVDF membrane, and

564 analyzed by immunoblotting with the corresponding antibodies (Fig. S6).

566 Overlay direct binding assay: Rhodopsin protein was expressed in HEK293 cells using

567 transient transfection (pcDNA3 rod opsin construct, a gift from Robert Lucas (Addgene

568 plasmid \# 109361, http://n2t.net/addgene:109361; RRID:Addgene_109361) [57] and

569 immunoprecipitated from the cell lysates using an anti-rhodopsin antibody (Abcam). In

570 parallel, rhodopsin was similarly immunoprecipitated from the mouse retinal lysate. The 
571 immunoprecipitated complexes were separated on SDS-PAGE gel and transferred to

572 PVDF membrane. The membrane was then probed by overlaying it with $5 \mu \mathrm{g}$ of

573 baculovirus-produced and purified recombinant full-length MYO1C FL or GFP-MYO1C-

$574790-1028$ (tail domain, also known as the cargo domain [13]) protein, by incubating at $4^{\circ} \mathrm{C}$

575 for $4 \mathrm{~h}$. Following incubation, the membranes were western blotted with MYO1C antibody

576 to detect the direct binding of MYO1C to the rhodopsin bands. The location of rhodopsin

577 on the membranes was marked by separately probing these membranes with an anti-

578 rhodopsin (1:500, Millipore Sigma) antibody (Fig. S6).

579

580 Quantitative Real Time-PCR: RNA was isolated from retinas of WT and Myo1c-KO 581 animals using Trizol reagent, and processed as described previously [55,70]. One 582 microgram of total RNA was reverse transcribed using the SuperScript II cDNA Synthesis

583 Kit (Invitrogen, Eugene, OR). Quantitative Real-Time PCR (qRT-PCR) was carried out 584 using SYBR green 1 chemistry (BioRad, Hercules, CA). Samples for qRT-PCR 585 experiments were assayed in triplicate using the BioRad CFX96 Q-PCR machine. Each 586 experiment was repeated twice ( $n=6$ reactions for each gene), using newly synthesized 587 cDNA.

589 Liver function tests using Alanine Aminotransferase (ALT) assays: To extract total 590 protein, liver tissue from WT or Myo1c-KO mice (pooled livers $n=4$ mice per genotype 591 respectively) were homogenized in RIPA buffer on ice and then centrifuged at 14,000 rpm 592 at $4^{\circ} \mathrm{C}$ for $10 \mathrm{~min}$. Supernatant was collected, and the protein concentration was 593 estimated using the Bio-Rad Protein Assay Dye Reagent (Sigma). $10 \mu$ l of liver lysate 
594 was transferred to 96-well plate and ALT was measured using a microplate-based ALT

595 activity assay kit (Pointe Scientific, Cat. A7526). Five biological replicates were used in

596 the assay.

597

Heart function tests using Echocardiographic (ECHO) analyses: Echocardiographic

$599(\mathrm{ECHO})$ analysis was performed on adult wildtype (WT) and Myo1c-KO animals ( $n=4$ per

600 genotype) at the MUSC Cardiology Core Facility. For ECHO experiments, mutant and

601 wild-type littermate controls were anesthetized in an induction chamber with 5\%

602 isoflurane in $100 \%$ oxygen. They were removed and placed on a warming table where

603 anesthesia was maintained via nose cone delivery of isoflurane ( $1 \%$ in $100 \%$ oxygen).

604 They were placed in the supine position, and the thoracic area was shaved. The limbs

605 were taped to the platform to restrict animal movement during echocardiography

606 acquisition. This also provided a connection to ECG leads embedded in the platform.

607 Sonography gel was applied to the chest and echocardiographic measurements of the

608 peristernal long axis and short axis of the heart were acquired to derive the systolic and

609 diastolic parameters of heart function. ECHO measurements were estimated using vevo

6102100 instrumentation.

611

612 Statistical Analysis: Data were expressed as means \pm standard deviation by ANOVA in

613 the Statistica 12 software (StatSoft Inc., Tulsa, Oklahoma, USA). Differences between

614 means were assessed by Tukey's honestly significant difference (HSD) test. $P$-values

615 below $0.05(P<0.05)$ were considered statistically significant. For western blot analysis,

616 relative intensities of each band were quantified (densitometry) using the Image $J$ 
617 software version 1.49 and normalized to the loading control $\beta$-actin. The qRT-PCR

618 analysis was normalized to $18 \mathrm{~S} \mathrm{RNA}$, and the $\Delta \Delta \mathrm{Ct}$ method was employed to calculate

619 fold changes. Data of qRT-PCR were expressed as mean \pm standard error of mean

620 (SEM). Statistical analysis was carried out using PRISM 8 software-GraphPad.

622 Data Availability

623 The authors declare that all data supporting the finding of this study are available within

624 this article and its supplementary information files or from the lead corresponding author

625 (G.P.L) upon request. The plasmids will be available from the lead corresponding author

626 (G.P.L) upon request.

628 Disclosure

629 All the authors declared no competing interests.

\section{Acknowledgements}

632 This work was supported by the National Institute of Health (NIH) grants, R21EY025034

633 and R01EY030889 to G.P.L.; 2R01DK087956-06A1, R56-DK116887-01A1, and

634 1R03TR003038-01 to D.N.; EY027013-02 to M.R.B.; and R01EY027355 to S.H. This

635 project was also supported in part by a DCl research grant (019898-001) and by a SCTR-

636 NIH/NCATS grant (5UL1TR001450) to G.P.L. The pCDNA3 Rod Opsin construct was a

637 gift from Dr. Robert Lucas (Addgene plasmid \# 109361; http://n2t.net/addgene:109361;

638 RRID:Addgene_109361). The authors thank George Robertson (Keyence Microscopes)

639 for the use of the Keyence BZ-X800 scope for semi-thin plastic sections, H\&E sections, 
640 and immunofluorescence imaging. The authors thank Dr. Don Rockey (MUSC) and Dr.

641 Seok-Hyung Kim (MUSC) for recommending suitable liver function tests and/or for

642 providing the ALT liver function kit. We also thank Dr. Linda McLoon (University of

643 Minnesota) for critical review of the manuscript.

645 Author Contributions

646 G.P.L. and D.N. designed the research studies and wrote the manuscript. G.P.L., D.N.,

647 B. Rohrer, M.R.B., S.H., H-J. K., R.M., and J.S. edited the manuscript. G.P.L., A.K.S., 648 M.R.B., R.D.M., E.O., D.N., E.A., B.R., S.W., S.H., and R.A.N. conducted experiments 649 and acquired data. A.K.S., G.P.L., M.R.B., S.W., S.H., J.S., R.D.M., R.A.N. and D.N., 650 analyzed and interpreted the data. M.R.B. and S.H. performed ERG and interpreted the 651 data. R.D.M and R.A.N. performed ECHO and/or interpreted the data. R.M., H-J. K., 652 R.A.N., R.D.M., M.R.B., J.S., S.H., B. Rohrer and J.H.L., supplied reagents, software, or 653 provided equipment for data analysis. All authors have read and agreed to the published 654 version of the manuscript.

\section{Figure Legends}

657 Fig. 1: MYO1C localizes to photoreceptors in mouse retina: Eyes from adult wild-type 658 (WT) and Myo1c-KO mice ( $n=8$ mice per genotype; 50:50 ratio of male and female) were 659 harvested and retina sections ( $n=5-7$ sections per eye) were immunostained with an anti660 MYO1C antibody (a-c), M-opsin antibody (b, c), followed by secondary (Alexa 488 or 661 Alexa 594) antibody staining. MYO1C (green fluorescence), M-Opsin (red fluorescence), 662 and DAPI or Hoechst (blue fluorescence). Figures in a-c are representative of retinal 663 sections ( $n=5-7$ sections per eye) imaged from $n=8$ animals per genotype. (b, c) Merge 
664 (orange) represents co-localization of MYO1C-488 (green) with M-Opsin-594 (red). RPE,

665 retinal pigmented epithelium; OS, outer segments; IS, inner segments; ONL, outer

666 nuclear layer. (a-c) Scale bar=50 $\mu \mathrm{m}$. (d) Total protein isolated from WT ( $n=4)$ and Myo1c-

$667 \mathrm{KO}(n=4)$ mouse retinas were pooled respectively and subjected to SDS-PAGE. Two

668 different concentrations of protein $(10 \mu \mathrm{g}$ and $20 \mu \mathrm{g})$ were used. Blots were then probed

669 with anti-Myo1c and Gapdh antibodies. Western blot analysis were repeated thrice.

670 Arrows indicate MYO1C protein band in retinal lysates of WT mice.

671

672 Fig. 2: Genetic deletion of Myo1c in mice results in decreased visual function: Dark-

673 adapted scotopic ERGs were recorded in response to increasing light intensities in

674 cohorts of control wild-type/WT (blue bars, blue-traces) and Myo1c-KO (red bars, red-

675 traces) mice of two month old $(\mathbf{a}, \mathbf{c})$, and six month old (b, d). Two-month-old Myo1c-KO

676 mice had lower dark-adapted $a$ - and $b$-wave amplitudes compared with controls (post-

677 hoc ANOVA: a-waves, * $p<0.0068$; $b$-waves, $p<0.0098$, n.s. not significant.), in particular

678 at higher light intensities (-40, -30, -20, -10, $0 \mathrm{~dB})$. Six-month-old Myo1c-knockout mice

679 had lower dark-adapted $a$ - and $b$-wave amplitudes compared with controls (post-hoc

680 ANOVA: a-waves, ${ }^{\star *} p<0.005$; $b$-waves, $\left.{ }^{\star \star} p<0.005\right)$, in particular at higher light intensities

$681(-40,-30,-20,-10,0 \mathrm{~dB})$. Photoreceptor cell responses (a-waves), which drive the $b$ -

682 waves, were equally affected in 6-months old Myo1c-KO animals (both reduced on

683 average between $38-45 \%$ of WT animals). Data are expressed as mean \pm S.E. (Myo1c-

684 KO mice and WT mice, $n=8$ per genotype and age-group; 50:50 ratio of male and female). 
Fig. 3: Immunohistochemical analysis of wild-type/WT and Myo1c-knockout mice retinas shows opsin trafficking defects: (a) Levels and localization of rhodopsin (Rho);

b, red/green medium wavelength cone opsin (M-opsin); c, short wavelength cone opsin

689 (S-opsin); d, PNA-488, were analyzed in two and six-months old WT and Myo1c-KO mice

690 retinas. Arrows in panel a highlight rhodopsin mislocalization to IS and cell bodies in

691 Myo1c-knockout mouse retinas. Images in panels a-d are representative of

692 immunostained retinal sections ( $n=5-7$ sections per eye) imaged from $n=8$ animals per

693 genotype and age-group (50:50 ratio of male and female). Scale bar=75 $\mu \mathrm{m}(\mathbf{a})$; Scale

694 bar=50 $\mu \mathrm{m}(\mathrm{b}, \mathbf{c}, \mathbf{d})$. OS, outer segments; IS, inner segments; ONL, outer nuclear layer;

695 INL, inner nuclear layer; OPL, outer plexiform layer.

697 Fig. 4: Immunohistochemical analysis of protein trafficking in photoreceptors of

698 wild-type/WT and Myo1c-knockout mice retinas: Levels and localization of (a) cone

699 arrestin (ARR), (b) Pde6b; (c) CNGA1; (d) Rod Arrestin (ARR1); and (e) G-protein

700 (Transducin), were analyzed in WT and Myo1c-KO mice retinas to evaluate protein

701 trafficking to photoreceptor OS. Red Arrows in panel a highlight cone photoreceptor

702 nuclei and OS in WT mouse retinas that were significantly reduced or shorter respectively

703 in Myo1C-KO animals (white arrows in a). Images in panels a-e are representative of

704 immunostained retinal sections ( $n=5-7$ sections per eye) imaged from $n=8$ animals per

705 genotype and age group (50:50 ratio of male and female). Panels a, $\mathbf{b}$, mice were 2-3

706 months of age. Panels c-e, mice were 3-4 months of age. (f) protein distribution (in \%) of

707 CNGA1, Rod ARR1, and Transducin, within the photoreceptor OS and IS, in light adapted

708 mice. For quantification of protein distribution within retinal layers, 5-7 retinal sections 
709 from each eye ( $n=8$ animals for each genotype) were analyzed using Image J. (g)

710 Representative western blot ( $n=3$ repeats) images of retinal proteins from 3-4 month old

711 WT and Myo1c-KO mice ( $n=2$ animals per genotype) showed no significant differences

712 in protein expression of key retinal genes among genotypes. OS, outer segments; IS,

713 inner segments; ONL, outer nuclear layer; INL, inner nuclear layer; OPL, outer plexiform

714 layer; IPL, inner plexiform layer.

715

716 Fig. 5: Histological analysis shows reduced photoreceptor OS lengths in Myo1c-

717 KO mice retinas: (a, b) Retinas from 2 and 6 month old WT and Myo1c-KO mice were

718 sectioned using an ultra-microtome and semi-thin plastic sections were obtained to

719 evaluate pathological consequences of MYO1C loss. Quantification of OS lengths from

720 H\&E sections (c, two month old mice; $\mathbf{d}, 6$ month old mice) and ONL thickness (e, two

721 month old mice; f, six months old mice) using "spider graph" morphometry. The OS

722 lengths and total number of layers of nuclei in the ONL of from H\&E sections through the

723 optic nerve (ON; $0 \mu \mathrm{m}$ distance from Optic Nerve and starting point) was measured at 12

724 locations around the retina, six each in the superior and inferior hemispheres, each

725 equally at $150 \mu \mathrm{m}$ distances. RPE, retinal pigmented epithelium; OS, outer segments; IS,

726 inner segments; ONL, outer nuclear layer; INL, inner nuclear layer; OPL, outer plexiform

727 layer; IPL, inner plexiform layer; GCL, ganglion cell layer. Retinal sections ( $n=5-7$ sections

728 per eye) from $n=8$ mice each genotype and time-point (50:50 ratio of male and female)

729 were analyzed. Two-way ANOVA with Bonferroni posttests compared Myo1c-KO mice

730 with WT in all segments. ${ }^{* *} \mathrm{p}<0.005$, for OS length in only 6 month old Myo1c-KO mice 
731 compared to WT mice; and n.s. (not significant) for ONL thickness in both 2 month and 6

732 month old Myo1c-KO animals, compared to WT mice). (a, b) Scale bar=100 $\mu \mathrm{m}$.

733

734 Fig. 6: Ultrastructural analysis of rods and cone photoreceptors using

735 transmission electron microscopy (TEM): Representative TEM images of rod

736 photoreceptors from two month (a) and six month (b) old WT and Myo1c-KO mice, are

737 presented. Representative images of cone photoreceptors from 2 month old WT (c) and

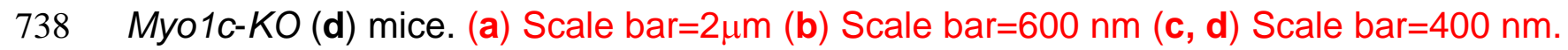

739 Data is representative of $n=6$ retinal sections per eye from $n=8$ mice per genotype and

740 time-point. (e) Rod OS (ROS) length in WT animals were measured and compared to

741 Myo1c-KO animals. (f) Cone OS (COS) length in WT animals were measured and

742 compared to Myo1c-KO animals. * $p<0.05 ;{ }^{* *} p<0.005$. RPE, retinal pigmented epithelium.

744 Fig. 7: Histological and immunohistochemical analysis of mice injected with PCIP:

745 The Myo1c specific inhibitor PCIP (pentachloropseudilin) or vehicle control DMSO was

746 injected retro-orbitally into the right eye of WT animals ( $n=2$ per treatment). (a) Levels and

747 localization of rhodopsin (Rho) and (b), red/green opsins (M-opsin). (c) Semi-thin plastic

748 sections of retina from mice were obtained to evaluate pathological consequences of

749 PCIP treatment. (d) Quantification of ONL thickness and (e) photoreceptor OS lengths

750 using "spider graph" morphometry, from H\&E sections. The OS length and total number

751 of layers of nuclei in the ONL of semi-thin plastic sections through the optic nerve (ON;

$7520 \mu \mathrm{m}$ distance and the starting point) was measure at 12 locations around the retina, six

753 each in the superior and inferior hemispheres, each equally at $150 \mu \mathrm{m}$ distance. Retinal 
754 sections ( $n=10-12$ sections per eye) from $n=2$ mice of each treatment group were

755 evaluated for ONL thickness and OS lengths. (a, b) Scale bar=50 $\mathrm{mm}$ (c) Scale bar=100

$756 \mu \mathrm{m}$. RPE, retinal pigmented epithelium; OS, outer segments; IS, inner segments; ONL,

757 outer nuclear layer; INL, inner nuclear layer; OPL, outer plexiform layer.

759 Fig. 8: Rhodopsin is a direct cargo for MY01C: (a) Mice retinal protein lysates were 760 isolated from Myo1c-KO and wild type (WT) mice (6 retinas pooled from $n=3$ mice per 761 genotype) and subjected to co-immunopreciptation analysis. Rhodopsin was co762 immunoprecipitated with MYO1C antibody (top panel). In a reciprocal manner, MYO1C 763 was co-immunoprecipated with Rhodopsin antibody (bottom panel). (b) Using Rhodopsin 764 antibody, Rhodopsin (RHO) was immunoprecipitated either from mice retinal lysates or 765 from HEK293 cells (transfected with pCDNA rhodopsin plasmid) where Rhodopsin was 766 overexpressed. The immunoprecipated Rhodopsin was separated using SDS-PAGE and 767 transferred to nitrocellulose membranes. The rhodopsin bound to nitrocellulose 768 membrane was then incubated with 5ug of purified recombinant active MYO1C-full length 769 (b) or MYO1C C-terminal cargo domain protein (c) generated from a baculovirus 770 expression system. To analyze if MYO1C binds to immobilized Rhodopsin, blots were 771 washed and western blotted with MYO1C antibody. A positive signal with MYO1C showed 772 direct binding of MYO1C to various Rhodopsin multimers (arrows) present in the retinal 773 lysate and overexpressed pCDNA3-Rhodopsin in HEK293 cells.

\section{Supplementary Figures}


Fig. S1: Myo1c targeting construct and Western blot analysis of MYO1C in systemic

tissues: (a) Schematic representation of the Myo1c targeting construct for generation of

the Myo1c-KO mouse line. (b) Western blot analysis confirmed MYO1C absence in

various systemic tissues of Myo1c-KO mice. Absence of MYO1C did not affect MYO1B

or MYO1E expression. Actin was used as the protein loading control. Representative

782 images from multiple western blots $(n=3)$ from $n=3$ animals per genotype.

783

Fig. S2: MY01C expression in mouse tissue by immunofluorescence: Expression of

MYO1C and ZO1 in systemic tissues, kidney (a), liver (b), and heart (c) of WT and Myo1c-

KO animals ( $n=3$ per genotype) by immunofluorescence. WT, wild type; KO, knockout.

Representative images from $n=3$ animals. (a, c) Scale bar=50 $\mu \mathrm{m}$; (b) Scale bar=75 $\mu \mathrm{m}$

Fig. S3: Quantification of OS lengths and distribution of Opsins in retinas of Myo1c-

791 quantified in two month old animals (a, b, and $\mathbf{c}$, respectively) and in six month old animals

792 (d, e, and $\mathbf{f}$, respectively). For quantification of rhodopsin distribution, 5-7 retinal sections

793 from each eye ( $n=8$ animals for each genotype and time-point) were analyzed using

794 Image $J$ or FIJI software. Mann-Whitney $U$ test was used for statistical analysis and

795 represented in Box-Whisker plots and considered significant ${ }^{*} p<0.05 ;{ }^{* *} p<0.005$.

797 Fig. S4: Immunohistochemical analysis of protein in RPE of wild-type/WT and

798 Myo1c-knockout mice retinas: The RPE specific protein STRA6 was used to evaluate 799 integrity of the RPE in both WT and Myo1c-KO mice. Representative images from $n=8$ 
800 animals (5-7 sections per eye) per genotype and age. Scale bar=50 $\mu \mathrm{m}$. RPE, retinal

801 pigmented epithelium; ONL, outer nuclear layer; INL, inner nuclear layer.

802

803 Fig. S5: Immunohistochemical and histological analysis of retinas from Cre+ mice:

804 Retinal histology, H\&E staining, and localization of rhodopsin (Rho), red/green cone opsin

805 (M-opsin) in WT (a) and Cre+ (b) mice. (a, b) Immunofluorescence Scale bar=50 $\mu$ m. $(\mathbf{a}$,

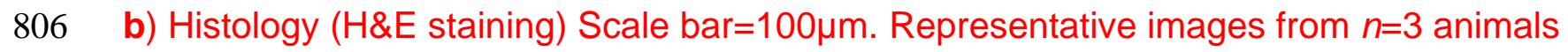

807 each genotype at 2-3 months of age (5-7 retinal sections per eye). RPE, retinal pigmented

808 epithelium; OS, outer segments; IS, inner segments; ONL, outer nuclear layer; OPL, outer

809 plexiform layer; INL, inner nuclear layer; IPL, inner plexiform layer; GCL, ganglion cell

810 layer.

811

812 Fig. S6: Schematic representation of MYO1C-Rhodopsin Co-IP binding

813 experiments from figure 8: Detailed flow chart of Co-IP binding experiments

814 representing figure 8 are shown.

816 Fig. S7: Representative tissue histology and functional analysis of systemic organs

817 from WT and Myo1c-KO animals: Systemic organs, liver (a), kidney (b), and heart (c),

818 of Myo1c and WT animals ( $n=4$ each genotype at 3-4 months of age) were sectioned and

819 stained with haematoxylin \& eosin (H\&E) to evaluate for systemic pathology of whole-

820 body MYO1C loss. Functional analysis of systemic organs using ALT liver function tests

821 ( $\left.\mathbf{a}^{\prime}\right)$, urine analysis for kidney proteinuria/albuminuria (b'), and heart function using

822 Echocardiography (c') was performed in Myo1c-KO animals and compared to WT 
823 controls. a', Liver function tests by Alanine Aminotransferase/ALT assay. No significant

824 change (n.s.) was found in total protein concentration or ALT activity of liver $(p>0.1)$ in

825 Myo1c-KO compared to WT mice. Five biological replicates were used for each assay

826 and statistical test used was one-tailed students $t$-test. b', approximately $20 \mu$ of urine

827 from Myo1c-KO and WT animals were electrophoresed on SDS-PAGE gels and stained

828 with Comassie blue. 5ug/ml BSA was used as positive control. BSA, bovine serum

829 albumin. c', representative images from WT and Myo1c-KO heart showing B-Mode long

830 axis and M-Mode short axis. $(\mathbf{a}, \mathbf{b}, \mathbf{c})$ Scale bar=50 $\mathrm{mm}$.

832 Fig. S8: Detailed Echocardiographic (ECHO) parameters in WT and Myo1c-KO

833 animals: Echocardiographic measurements was taken using the vevo 2100 ultrasound

834 imaging system, to access cardiac function among genotypes. (a) Left-ventricle at end-

835 diastole, Posterior wall thickness, and Septal wall thickness; (b) Left-ventricle (LV) Mass;

836 (c) Stroke volume (SV); (d) Left-ventricle ejection fraction (EF); (e) Left-ventricle end-

837 diastolic volume (EDV); and (f) tabular summary of ECHO values from $n=4$

838 animals/genotype at 3-4 months of age. By t-test, there were no statistically significant

839 differences between the two genotypes. Values presented as MeanıSEM.

841 Fig. S9: qPCR analysis of various MYO1C family members in mice retina: Retinas

842 from WT and Myo1c-KO mice were isolated and processed for qPCR analysis using

843 specific primers for various Myo1c family members including Myo1b, $d$, e and $f$. qPCR

844 analysis was performed in triplicates for each sample and repeated thrice with freshly

845 synthesized cDNA for each repeat experiment. 


\section{References}

847

848

849

850

851

852

853

854

855

856

857

858

859

860

861

862

863

864

865

866

867

868

869

870

871

872

873

874

875

876

877

878

879

880

881

882

883

884

885

886

887

888

889

890

1. Baehr, W., et al., The function of guanylate cyclase 1 and guanylate cyclase 2 in rod and cone photoreceptors. J Biol Chem, 2007. 282(12): p. 8837-47.

2. Coffey, P.J., et al., Complement factor $H$ deficiency in aged mice causes retinal abnormalities and visual dysfunction. Proc Natl Acad Sci U S A, 2007. 104(42): p. 16651-6.

3. Fan, J., et al., Rpe65-/- and Lrat-/- mice: comparable models of leber congenital amaurosis. Invest Ophthalmol Vis Sci, 2008. 49(6): p. 2384-9.

4. Grossman, G.H., et al., Immunocytochemical evidence of Tulp1-dependent outer segment protein transport pathways in photoreceptor cells. Exp Eye Res, 2011. 93(5): p. 658-68.

5. Hüttl, S., et al., Impaired channel targeting and retinal degeneration in mice lacking the cyclic nucleotide-gated channel subunit CNGB1. J Neurosci, 2005. 25(1): p. 130-8.

6. Zhang, $\mathrm{H}$., et al., Trafficking of membrane-associated proteins to cone photoreceptor outer segments requires the chromophore 11-cis-retinal. J Neurosci, 2008. 28(15): p. 4008-14.

7. Pearring, J.N., et al., Protein sorting, targeting and trafficking in photoreceptor cells. Prog Retin Eye Res, 2013. 36: p. 24-51.

8. Zhang, T., et al., Cone opsin determines the time course of cone photoreceptor degeneration in Leber congenital amaurosis. Proc Natl Acad Sci U S A, 2011. 108(21): p. 8879-84.

9. Karan, S., et al., A model for transport of membrane-associated phototransduction polypeptides in rod and cone photoreceptor inner segments. Vision Research, 2008. 48(3): p. 442-452.

10. Abdelkader, E., et al., Severe retinal degeneration at an early age in Usher syndrome type $1 B$ associated with homozygous splice site mutations in MYO7A gene. Saudi J Ophthalmol, 2018. 32(2): p. 119-125.

11. Cheng, L., et al., Identification of a novel MYO7A mutation in Usher syndrome type 1. Oncotarget, 2018. 9(2): p. 2295-2303.

12. Arif, E., et al., Motor protein Myo1c is a podocyte protein that facilitates the transport of slit diaphragm protein Neph1 to the podocyte membrane. Mol Cell Biol, 2011. 31(10): p. 2134-50.

13. Nakamori, Y., et al., Myosin motor Myo1c and its receptor NEMO/IKK-gamma promote TNF-alpha-induced serine307 phosphorylation of IRS-1. J Cell Biol, 2006. 173(5): p. 665-71.

14. Woolner, S. and W.M. Bement, Unconventional myosins acting unconventionally. Trends Cell Biol, 2009. 19(6): p. 245-52.

15. Bownds, D., Site of attachment of retinal in rhodopsin. Nature, 1967. 216(5121): p. 1178-81.

16. Palczewski, K., et al., Crystal structure of rhodopsin: A G protein-coupled receptor. Science, 2000. 289(5480): p. 739-45.

17. Wald, G., J. Durell, and C.C. St George, The light reaction in the bleaching of rhodopsin. Science, 1950. 111(2877): p. 179-81. 
891

892

893

894

895

896

897

898

899

900

901

902

903

904

905

906

907

908

909

910

911

912

913

914

915

916

917

918

919

920

921

922

923

924

925

926

927

928

929

930

931

932

933

934

935

936

18. Wald, G. and R. Hubbard, The Synthesis of Rhodopsin from Vitamin A(1). Proc Natl Acad Sci U S A, 1950. 36(2): p. 92-102.

19. Wang, J.K., J.H. McDowell, and P.A. Hargrave, Site of attachment of 11-cis-retinal in bovine rhodopsin. Biochemistry, 1980. 19(22): p. 5111-7.

20. Walsh, T., et al., From flies' eyes to our ears: mutations in a human class III myosin cause progressive nonsyndromic hearing loss DFNB30. Proc Natl Acad Sci U S A, 2002. 99(11): p. 7518-23.

21. Rehman, A.U., et al., Mutational Spectrum of MYO15A and the Molecular Mechanisms of DFNB3 Human Deafness. Human mutation, 2016. 37(10): p. 9911003.

22. Samuels, I.S., et al., Myosin 6 is required for iris development and normal function of the outer retina. Invest Ophthalmol Vis Sci, 2013. 54(12):p. 7223-33.

23. Adamek, N., M.A. Geeves, and L.M. Coluccio, Myo1c mutations associated with hearing loss cause defects in the interaction with nucleotide and actin. Cell Mol Life Sci, 2011. 68(1): p. 139-50.

24. Lin, T., et al., A hearing loss-associated myo1c mutation (R156W) decreases the myosin duty ratio and force sensitivity. Biochemistry, 2011. 50(11): p. 1831-8.

25. Skeie, J.M. and V.B. Mahajan, Proteomic interactions in the mouse vitreous-retina complex. PLoS One, 2013. 8(11): p. e82140.

26. Boguslavsky, S., et al., Myo1c binding to submembrane actin mediates insulininduced tethering of GLUT4 vesicles. Mol Biol Cell, 2012. 23(20): p. 4065-78.

27. Bose, A., et al., Unconventional myosin Myo1c promotes membrane fusion in a regulated exocytic pathway. Mol Cell Biol, 2004. 24(12): p. 5447-58.

28. Fan, Y., et al., Myo1c facilitates G-actin transport to the leading edge of migrating endothelial cells. J Cell Biol, 2012. 198(1): p. 47-55.

29. Arif, E., et al., The motor protein Myo 1c regulates transforming growth factor-betasignaling and fibrosis in podocytes. Kidney Int, 2019.

30. Crosson, C.E., et al., Inhibition of histone deacetylase protects the retina from ischemic injury. Invest Ophthalmol Vis Sci, 2010. 51(7): p. 3639-45.

31. Husain, S., D.E. Potter, and C.E. Crosson, Opioid receptor-activation: retina protected from ischemic injury. Invest Ophthalmol Vis Sci, 2009. 50(8): p. 3853-9.

32. Krebs, W. and H. Kühn, Structure of isolated bovine rod outer segment membranes. Exp Eye Res, 1977. 25(5): p. 511-26.

33. Pearring, J.N., et al., Protein sorting, targeting and trafficking in photoreceptor cells. Progress in retinal and eye research, 2013. 36: p. 24-51.

34. Calvert, P.D., et al., Light-driven translocation of signaling proteins in vertebrate photoreceptors. Trends Cell Biol, 2006. 16(11): p. 560-8.

35. Brandstaetter, $\mathrm{H}$., et al., Loss of functional MYO1C/myosin 1c, a motor protein involved in lipid raft trafficking, disrupts autophagosome-lysosome fusion. Autophagy, 2014. 10(12): p. 2310-23.

36. Mustafi, D., et al., Photoreceptor phagocytosis is mediated by phosphoinositide signaling. FASEB journal : official publication of the Federation of American Societies for Experimental Biology, 2013. 27(11): p. 4585-4595.

37. Lopes, V.S., et al., The Usher $1 B$ protein, MYO7A, is required for normal localization and function of the visual retinoid cycle enzyme, RPE65. Human molecular genetics, 2011. 20(13): p. 2560-2570. 
38. Chinthalapudi, K., et al., Mechanism and specificity of pentachloropseudilinmediated inhibition of myosin motor activity. J Biol Chem, 2011 . 286(34): p. 297008.

39. Gupta, P., et al., Myosin-1 inhibition by PCIP affects membrane shape, cortical actin distribution and lipid droplet dynamics in early Zebrafish embryos. PLoS One, 2017. 12(7): p. e0180301.

40. Kittelberger, N., et al., The role of myosin $1 \mathrm{c}$ and myosin $1 \mathrm{~b}$ in surfactant exocytosis. J Cell Sci, 2016. 129(8): p. 1685-96.

41. Martin, R., et al., Total synthesis of pentabromo- and pentachloropseudilin, and synthetic analogues--allosteric inhibitors of myosin ATPase. Angew Chem Int Ed Engl, 2009. 48(43): p. 8042-6.

42. Young, R.W., The renewal of photoreceptor cell outer segments. J Cell Biol, 1967. 33(1): p. 61-72.

43. Young, R.W. and B. Droz, The renewal of protein in retinal rods and cones. J Cell Biol, 1968. 39(1): p. 169-84.

44. Findlay, J.B. and D.J. Pappin, The opsin family of proteins. Biochem J, 1986. 238(3): p. 625-42.

45. Lobo, G.P., et al., Involvement of Endoplasmic Reticulum Stress in TULP1 Induced Retinal Degeneration. PLoS One, 2016. 11(3): p. e0151806.

46. Beech, P.L., et al., Localization of kinesin superfamily proteins to the connecting cilium of fish photoreceptors. J Cell Sci, 1996. 109 ( Pt 4): p. 889-97.

47. Muresan, V., et al., Evidence for kinesin-related proteins associated with the axoneme of retinal photoreceptors. Exp Eye Res, 1997. 64(6): p. 895-903.

48. Chaitin, M.H. and N. Coelho, Immunogold localization of myosin in the photoreceptor cilium. Invest Ophthalmol Vis Sci, 1992. 33(11): p. 3103-8.

49. Williams, D.S., M.A. Hallett, and K. Arikawa, Association of myosin with the connecting cilium of rod photoreceptors. J Cell Sci, 1992. 103 ( Pt 1): p. 183-90.

50. Mattapallil, M.J., et al., The Rd8 mutation of the Crb1 gene is present in vendor lines of C57BL/6N mice and embryonic stem cells, and confounds ocular induced mutant phenotypes. Invest Ophthalmol Vis Sci, 2012. 53(6): p. 2921-7.

51. Chung, C.L., et al., Pentachloropseudilin Inhibits Transforming Growth Factor- $\beta$ (TGF- $\beta$ ) Activity by Accelerating Cell-Surface Type II TGF- $\beta$ Receptor Turnover in Target Cells. Chembiochem, 2018. 19(8): p. 851-864.

52. Iuliano, O., et al., Myosin $1 \mathrm{~b}$ promotes axon formation by regulating actin wave propagation and growth cone dynamics. Journal of Cell Biology, 2018. 217(6): p. 2033-2046.

53. Cota Teixeira, S., et al., Pentachloropseudilin Impairs Angiogenesis by Disrupting the Actin Cytoskeleton, Integrin Trafficking and the Cell Cycle. Chembiochem, 2019. 20(18): p. 2390-2401.

54. Gupta, P., et al., Myosin $1 E$ localizes to actin polymerization sites in lamellipodia, affecting actin dynamics and adhesion formation. Biol Open, 2013. 2(12): p. 128899.

55. Rozbicki, E., et al., Myosin-II-mediated cell shape changes and cell intercalation contribute to primitive streak formation. Nat Cell Biol, 2015. 17(4): p. 397-408.

56. Prospéri, M.T., et al., Myosin $1 b$ functions as an effector of EphB signaling to control cell repulsion. J Cell Biol, 2015. 210(2): p. 347-61. 
983 57. Lobo, G.P., et al., The exocyst is required for photoreceptor ciliogenesis and retinal 984 development. J Biol Chem, 2017. 292(36): p. 14814-14826.

985 58. Husain, S., Delta Opioids: Neuroprotective Roles in Preclinical Studies. J Ocul

986 Pharmacol Ther, 2018. 34(1-2): p. 119-128.

987 59. Husain, S., et al., PI3K/Akt Pathway: A Role in delta-Opioid Receptor-Mediated 988 RGC Neuroprotection. Invest Ophthalmol Vis Sci, 2017. 58(14): p. 6489-6499. 


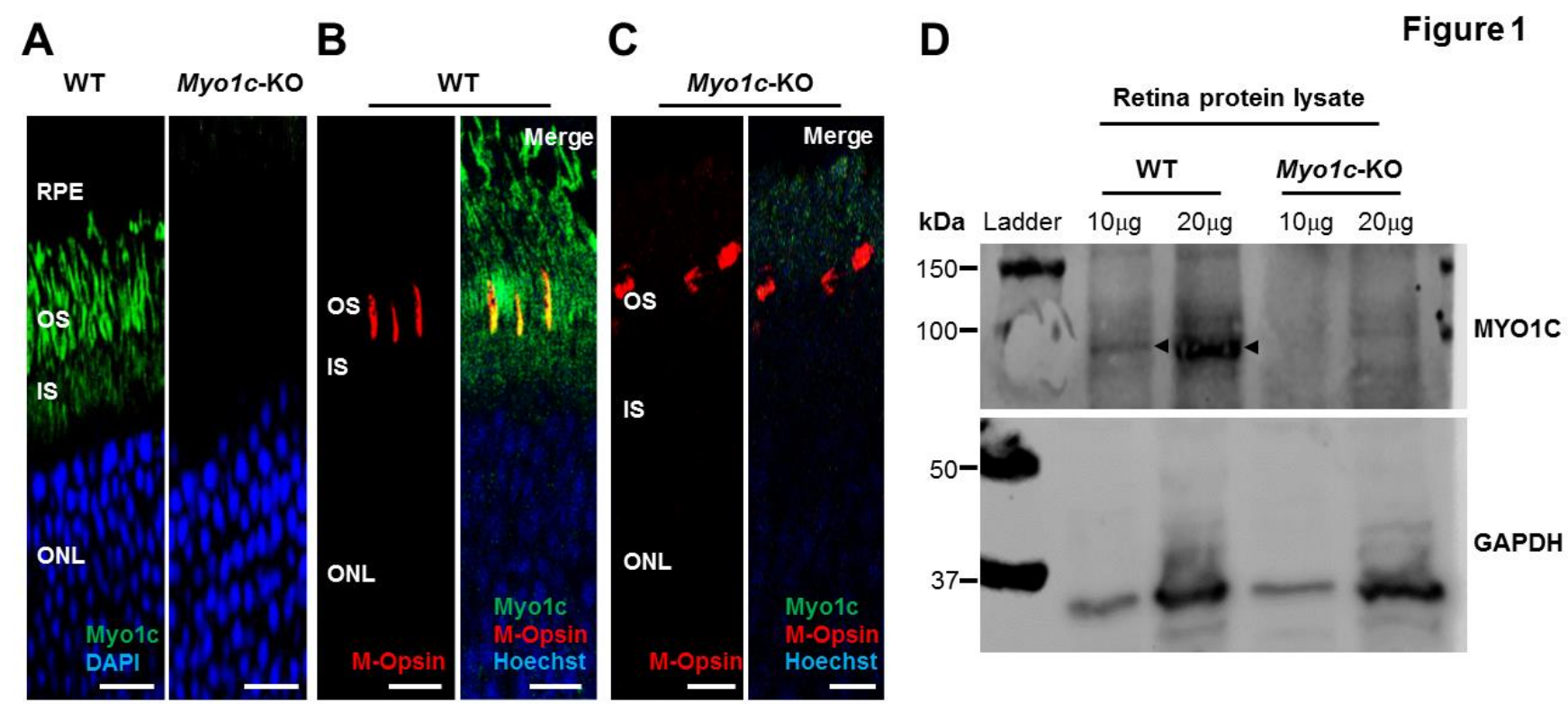



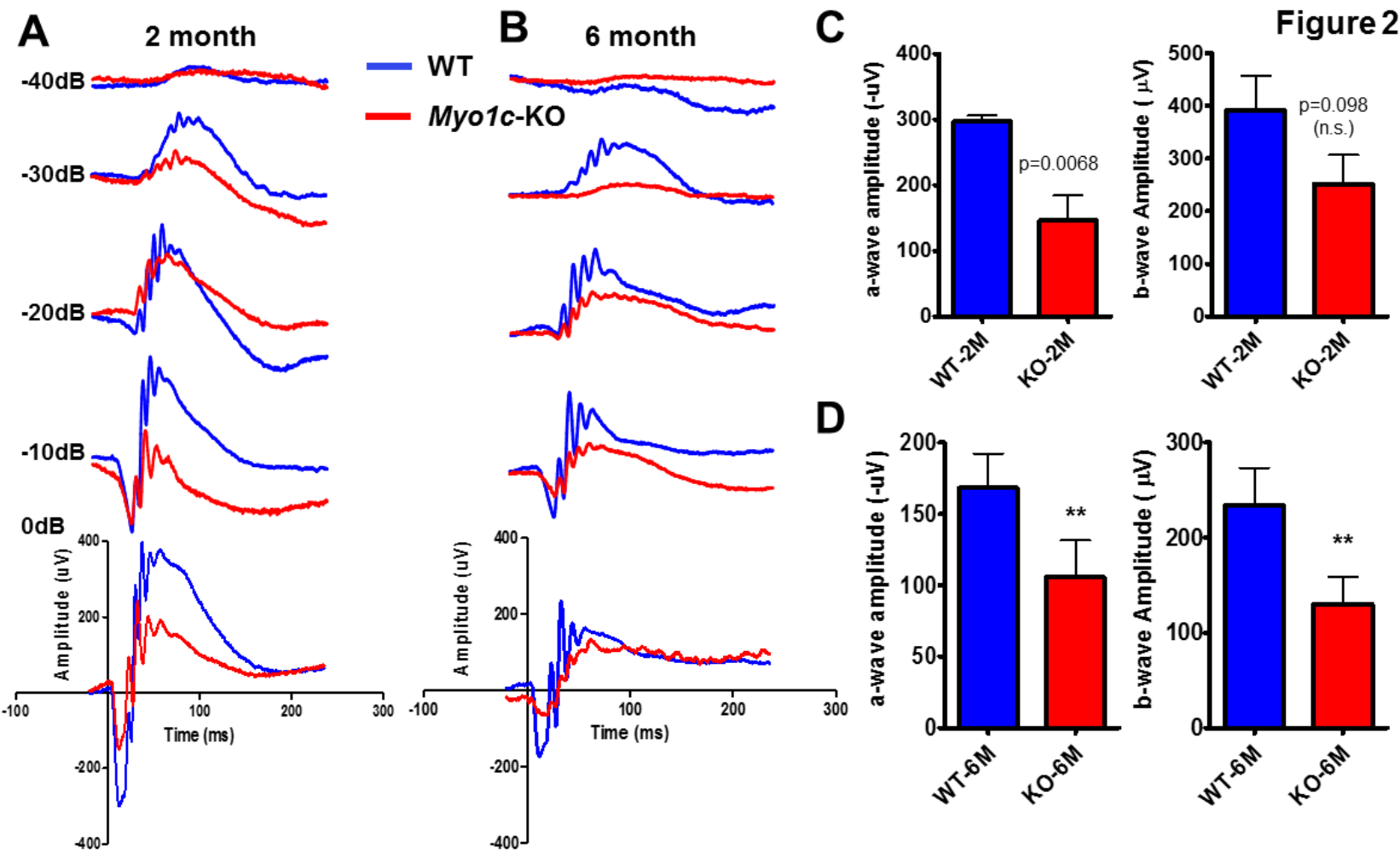

1059

1060

1061

1062

1063

1064

1065

1066

1067

1068

1069

1070

1071

1072

1073

1074

1075

1076

1077

1078

1079

1080

1081 
bioRxiv preprint doi: https://doi.org/10.1101/2020.06.02.129890; this version posted January 15,2021 . The copyright holder for this preprint (which was not certified by peer review) is the author/funder, who has granted bioRxiv a license to display the preprint in perpetuity. It is made available under aCC-BY-NC-ND 4.0 International license.

1082

1083

1084

1085

1086

1087

1088

1089

1090

1091

1092

1093

1094

1095

1096

1097

1098

1099

1100

1101

1102

1103

1104

1105

1106

1107
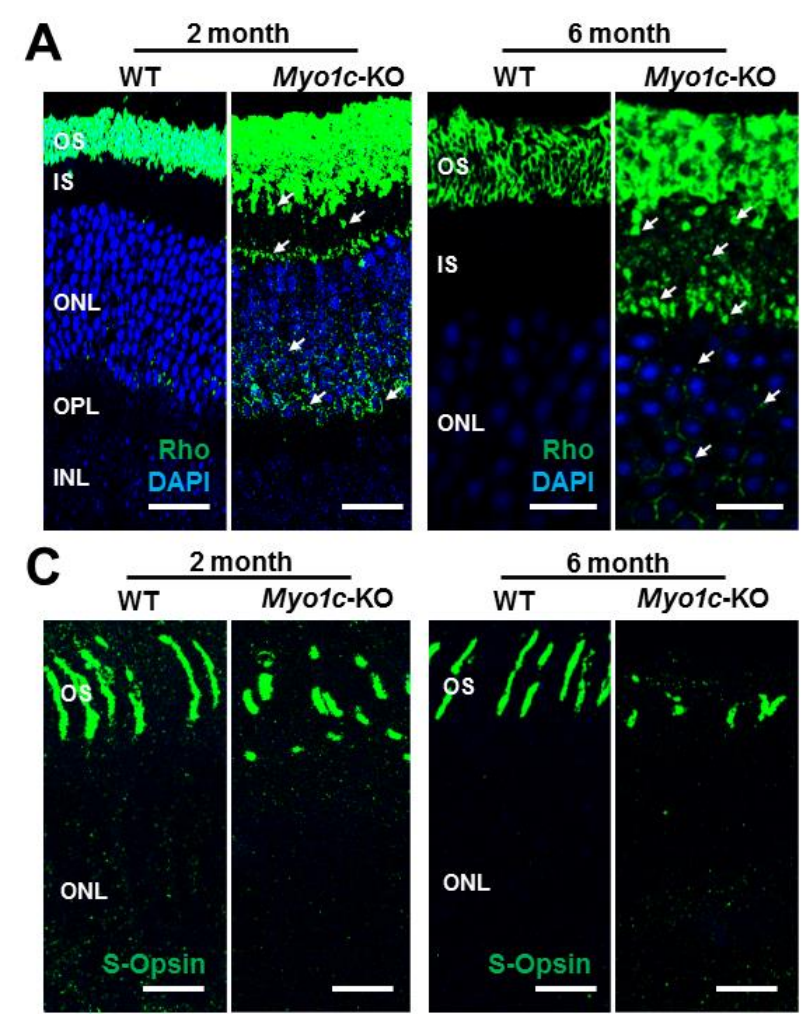
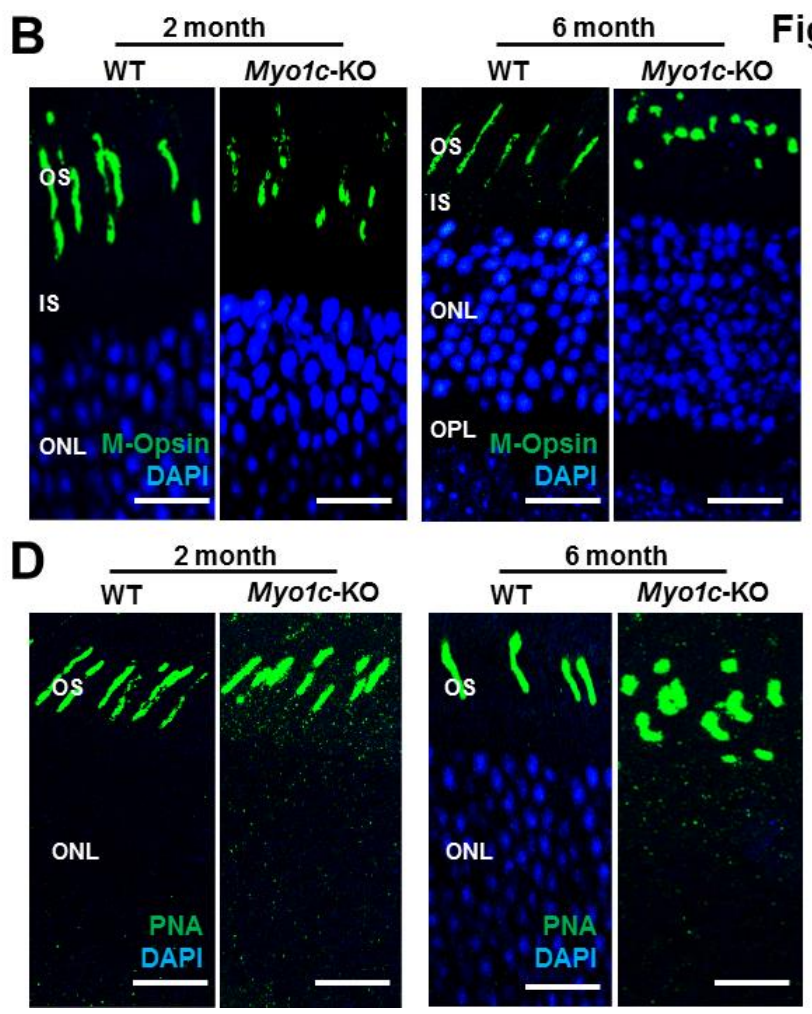

Figure 3 
bioRxiv preprint doi: https://doi.org/10.1101/2020.06.02.129890; this version posted January 15,2021 . The copyright holder for this preprint (which was not certified by peer review) is the author/funder, who has granted bioRxiv a license to display the preprint in perpetuity. It is made available under aCC-BY-NC-ND 4.0 International license.
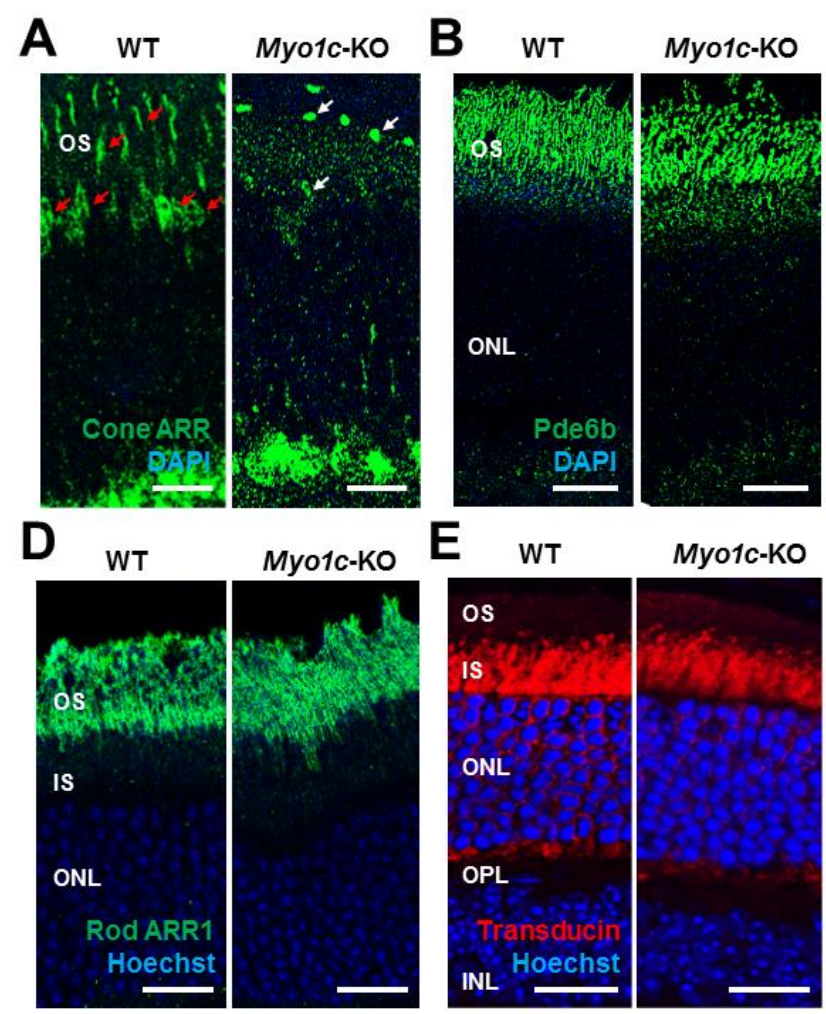
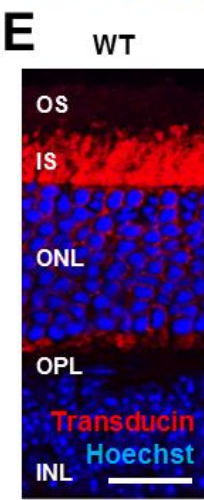
Myo1c-Ko

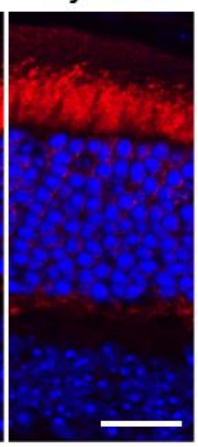

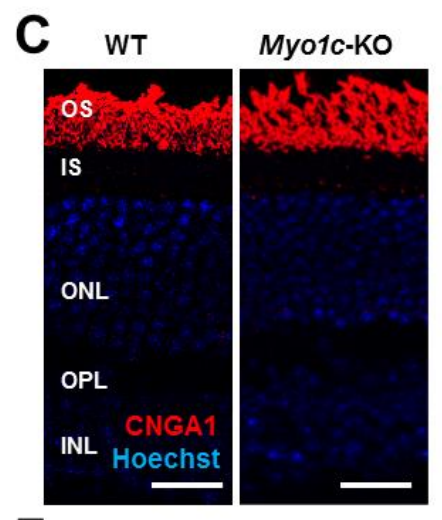

$F$

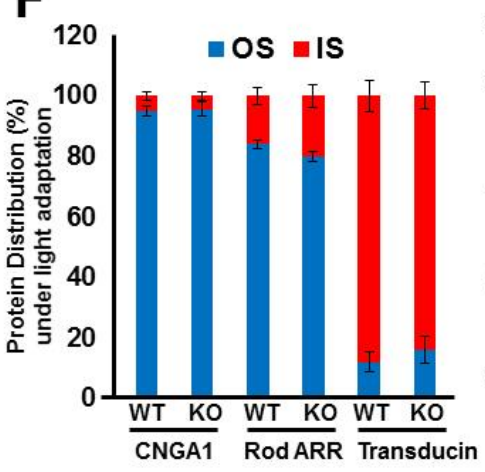

G

Figure 4
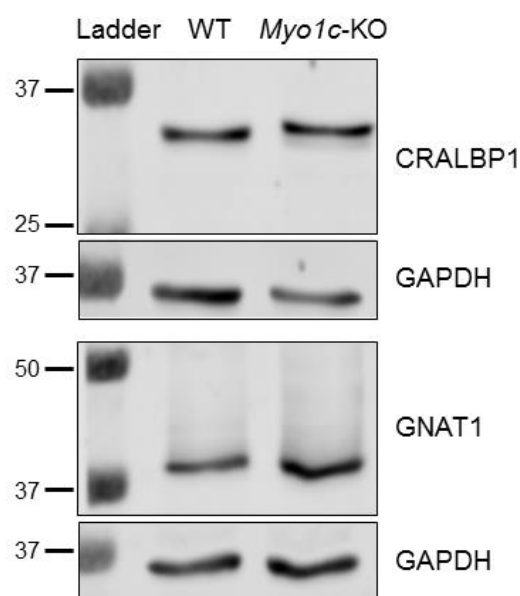

GNAT1

GAPDH

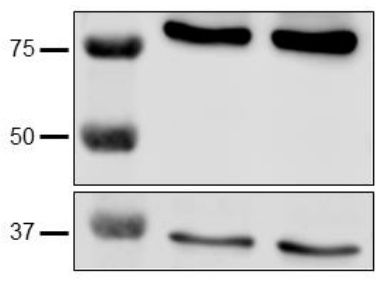

PKC 
bioRxiv preprint doi: https://doi.org/10.1101/2020.06.02.129890; this version posted January 15,2021 . The copyright holder for this preprint (which was not certified by peer review) is the author/funder, who has granted bioRxiv a license to display the preprint in perpetuity. It is made available under aCC-BY-NC-ND 4.0 International license.

A 2 month B

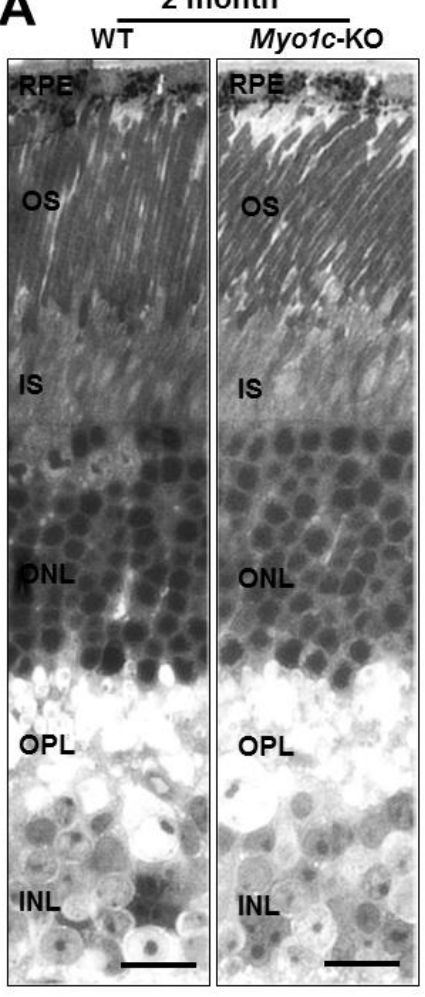

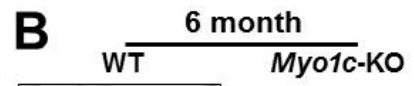

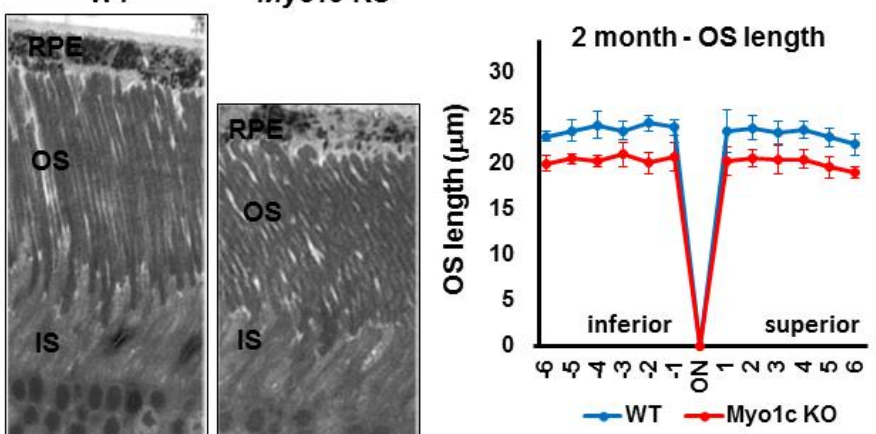

D

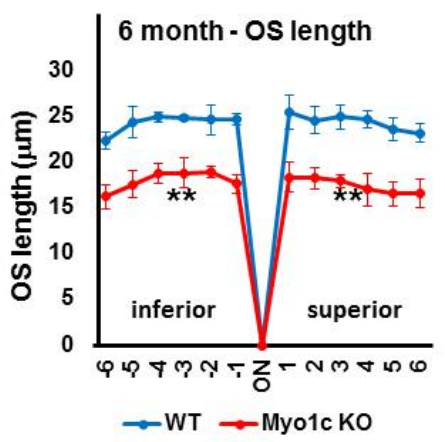

E

Figure 5

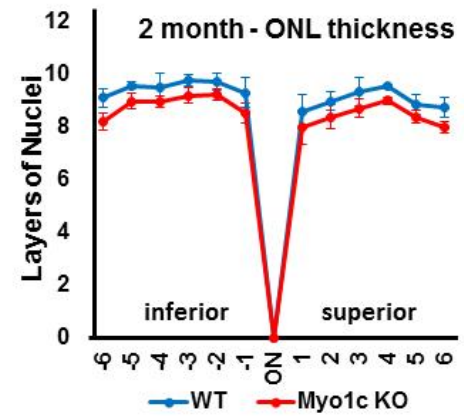

F

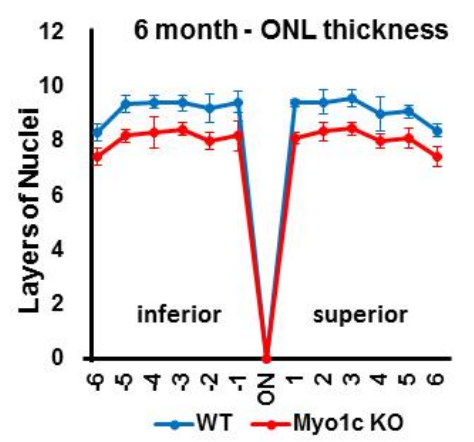


bioRxiv preprint doi: https://doi.org/10.1101/2020.06.02.129890; this version posted January 15,2021 . The copyright holder for this preprint (which was not certified by peer review) is the author/funder, who has granted bioRxiv a license to display the preprint in perpetuity. It is made available under aCC-BY-NC-ND 4.0 International license.

I
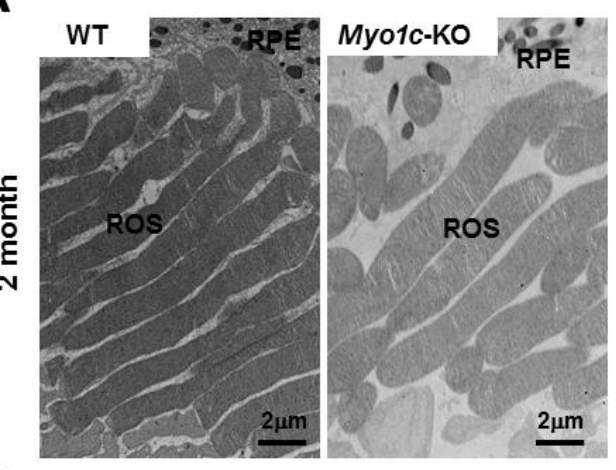

\}

1172

1173

1174

1175

1176

1177

1178

1179

1180

1181

1182

1183

1184

1185

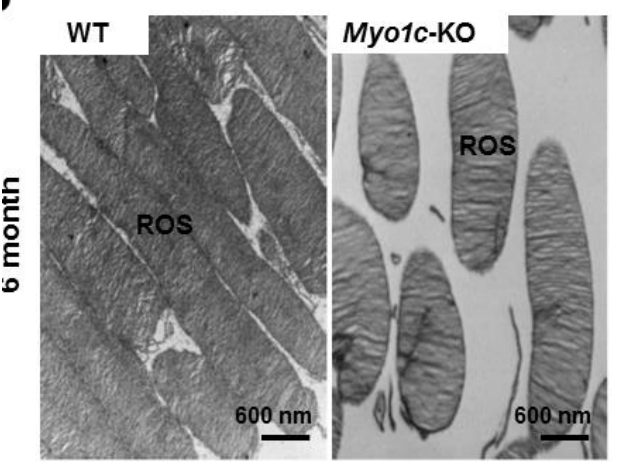

C

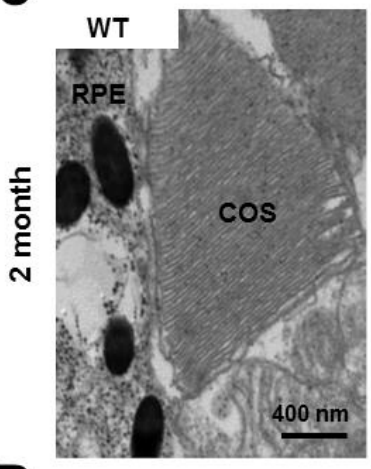

D

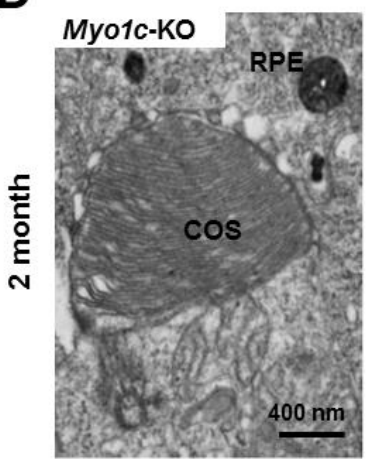

E Rod OS length

a 2 months $W T$

2 months Myo1c-KO

a 6 months WT

6 months Myo1c-Ko

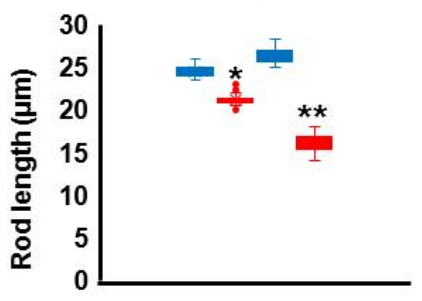

F

-WT $=$ Myo1c-Ko

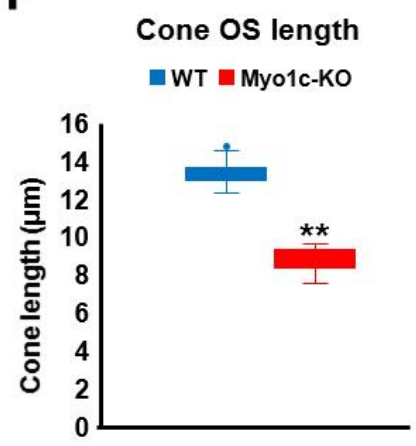

Figure 6 
bioRxiv preprint doi: https://doi.org/10.1101/2020.06.02.129890; this version posted January 15,2021 . The copyright holder for this preprint (which was not certified by peer review) is the author/funder, who has granted bioRxiv a license to display the preprint in perpetuity. It is made available under aCC-BY-NC-ND 4.0 International license.

A

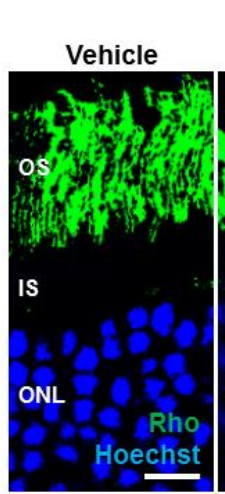

B

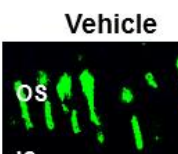

IS

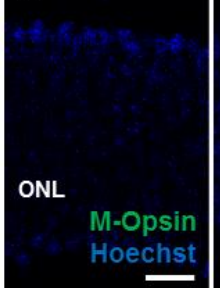

\section{Left Eye}

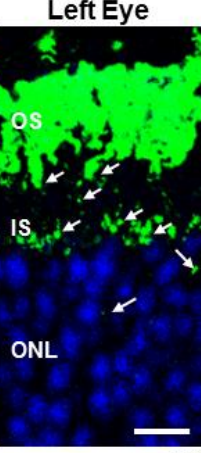

PCIP

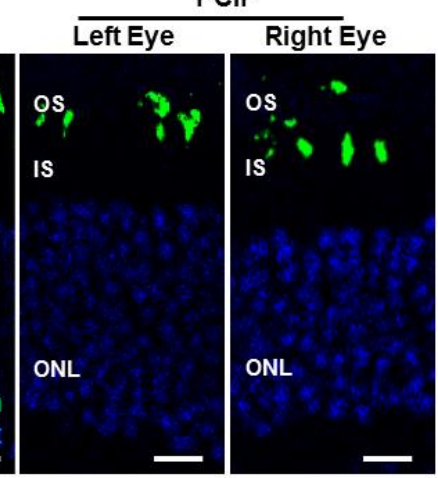

C

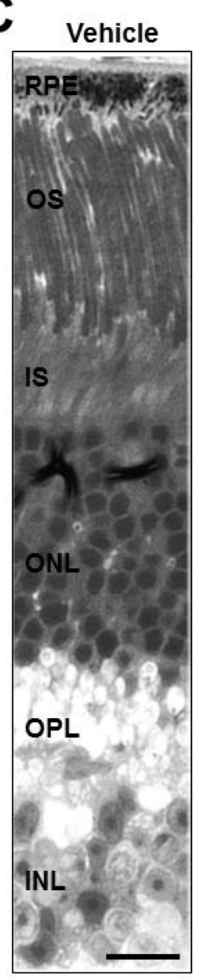

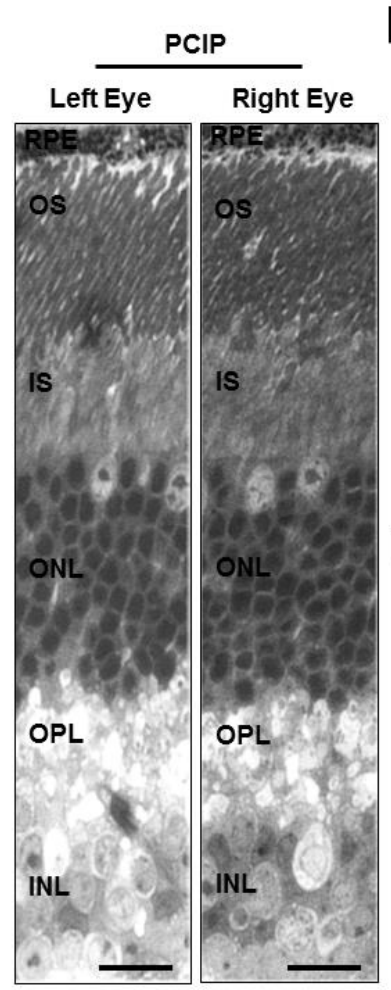

Figure 7

D

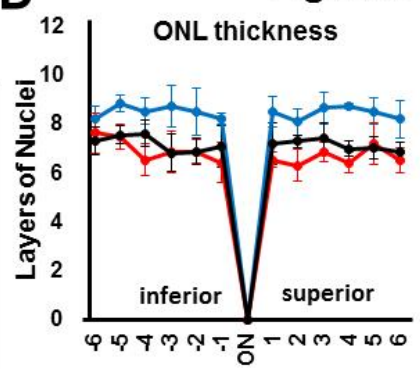

$\rightarrow$ WT-Vehicle

$\rightarrow$ WT-PCIP-Right Eye
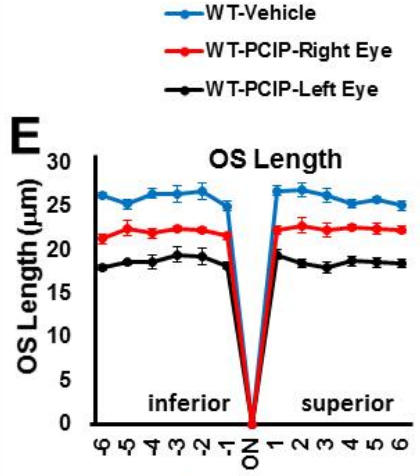

$\rightarrow$ WT-Vehicle

$\rightarrow$ WT-PCIP-Right Eye

$\rightarrow$ WT-PCIP-Left Eye 
A

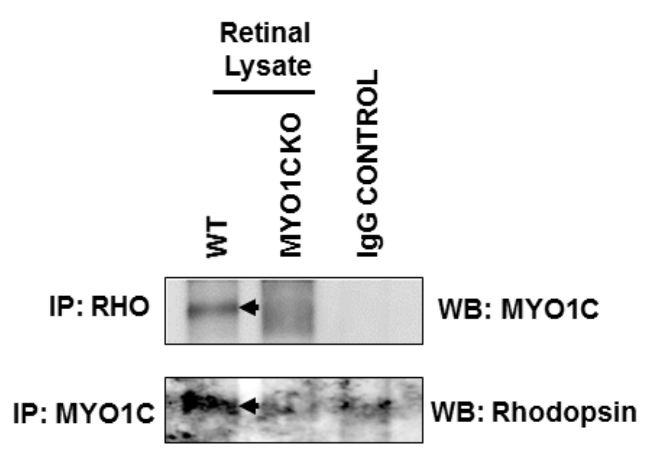

Retinal

Lysate

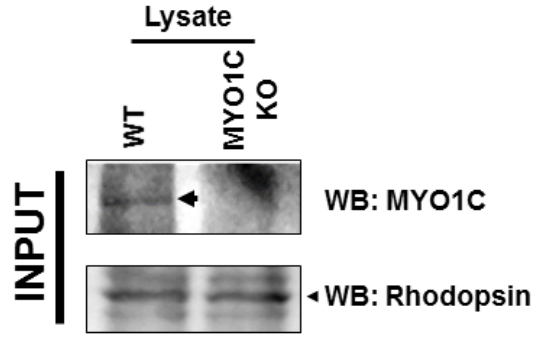

\section{B}

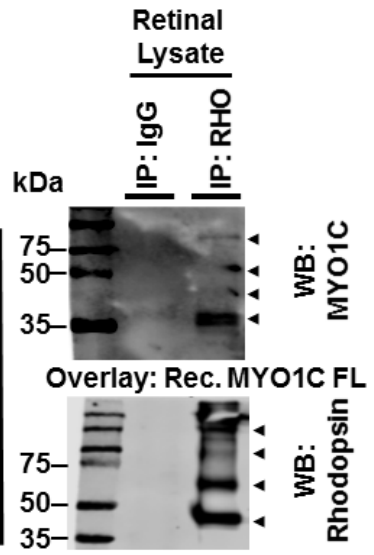

kDa

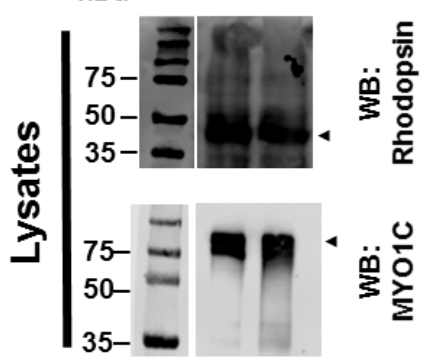

C

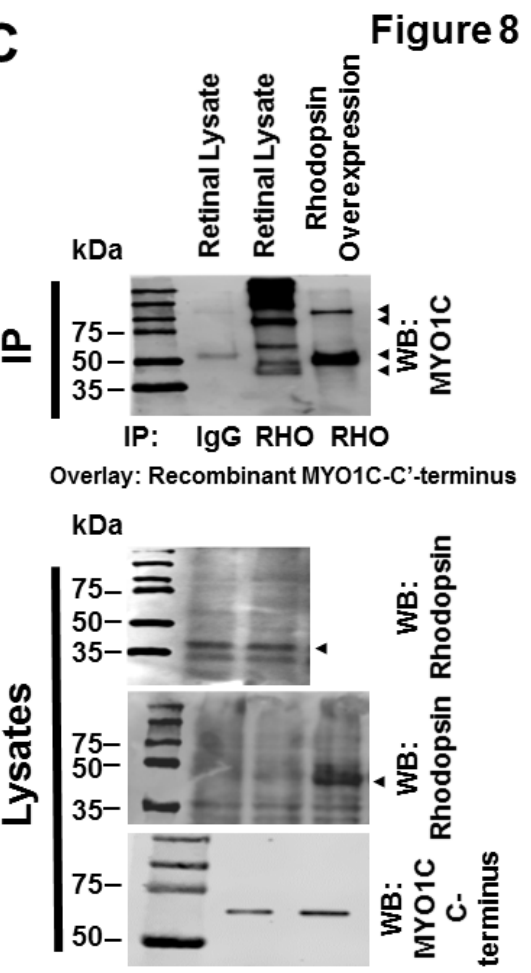


bioRxiv preprint doi: https://doi.org/10.1101/2020.06.02.129890; this version posted January 15, 2021. The copyright holder for this preprint (which was not certified by peer review) is the author/funder, who has granted bioRxiv a license to display the preprint in perpetuity. It is made available under aCC-BY-NC-ND 4.0 International license.

A

Myo1c Targeting Construct

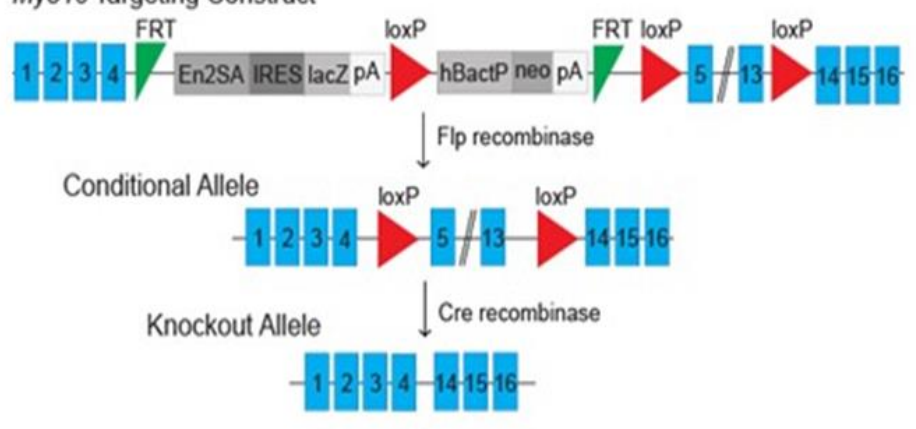

B

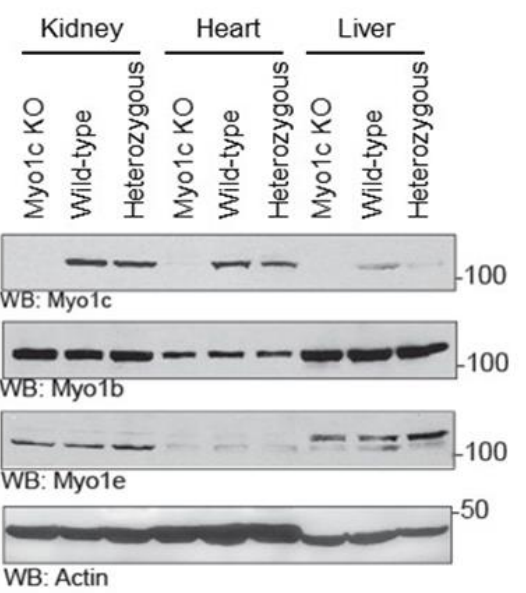


bioRxiv preprint doi: https://doi.org/10.1101/2020.06.02.129890; this version posted January 15,2021 . The copyright holder for this preprint (which was not certified by peer review) is the author/funder, who has granted bioRxiv a license to display the preprint in perpetuity. It is made available under aCC-BY-NC-ND 4.0 International license.
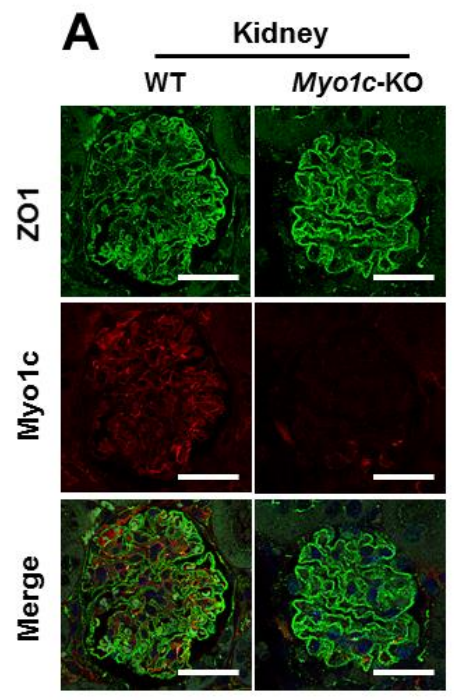

B
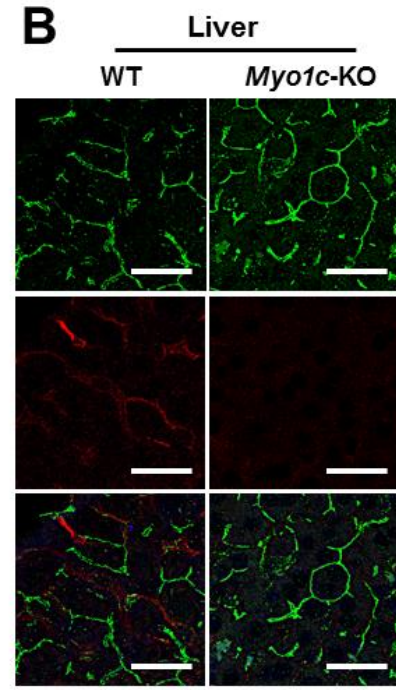

C WT

Figure S2

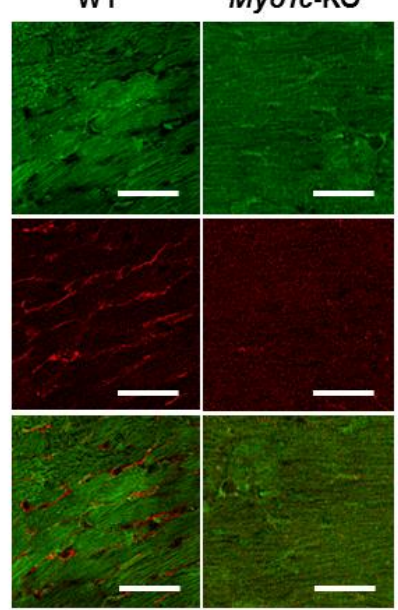



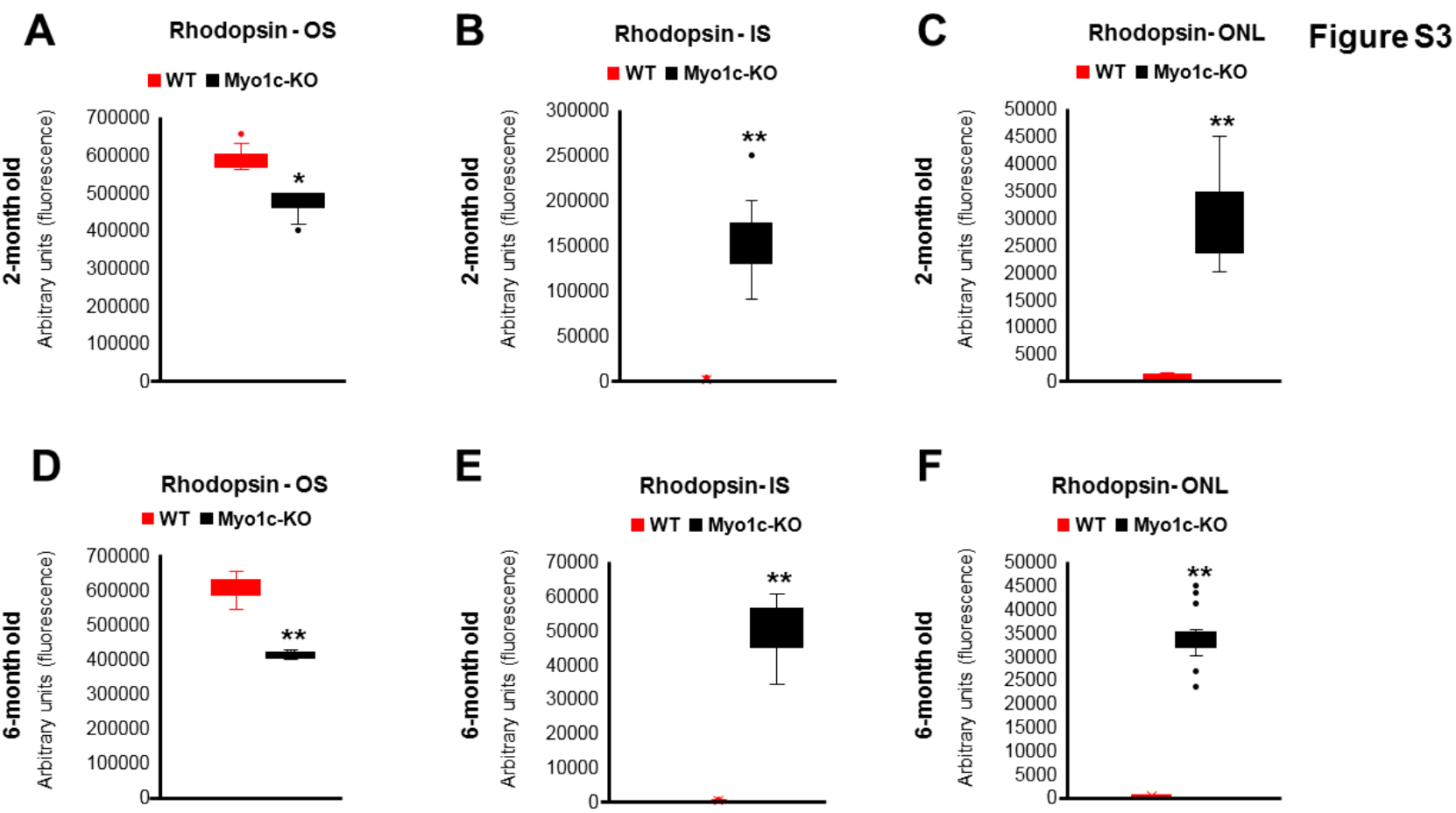

\section{$\mathbf{F}$}

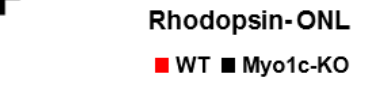

1292

1293

1294

1295

1296

1297

1298

1299

1300

1301

1302

1303

1304

1305

1306

1307

1308

1309

1310

1311

1312

1313 

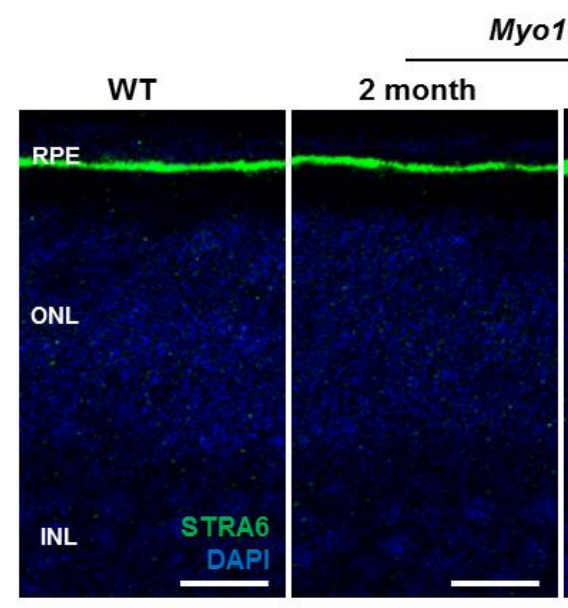

Figure S4

1314

1315

1316

1317

1318

1319

1320

1321

1322

1323

1324

1325

1326

1327

1328

1329

1330

1331

1332

1333

1334

1335

1336

1337

1338

1339 
bioRxiv preprint doi: https://doi.org/10.1101/2020.06.02.129890; this version posted January 15,2021 . The copyright holder for this preprint (which was not certified by peer review) is the author/funder, who has granted bioRxiv a license to display the preprint in perpetuity. It is made available under aCC-BY-NC-ND 4.0 International license.
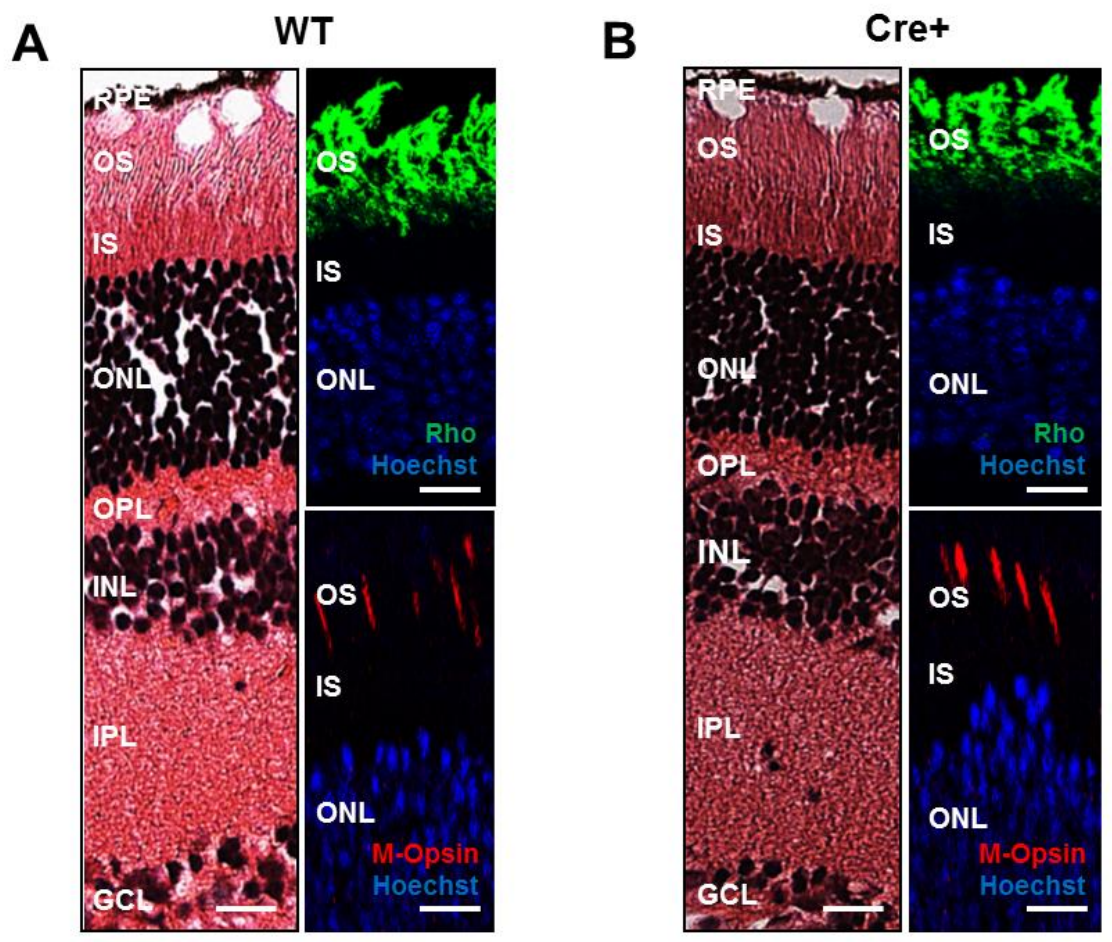

Figure 55

1343

1344

1345

1346

1347

1348

1349

1350

1351 
bioRxiv preprint doi: https://doi.org/10.1101/2020.06.02.129890; this version posted January 15, 2021. The copyright holder for this preprint (which was not certified by peer review) is the author/funder, who has granted bioRxiv a license to display the preprint in perpetuity. It is made available under aCC-BY-NC-ND 4.0 International license.

Figure $\mathbf{5 6}$

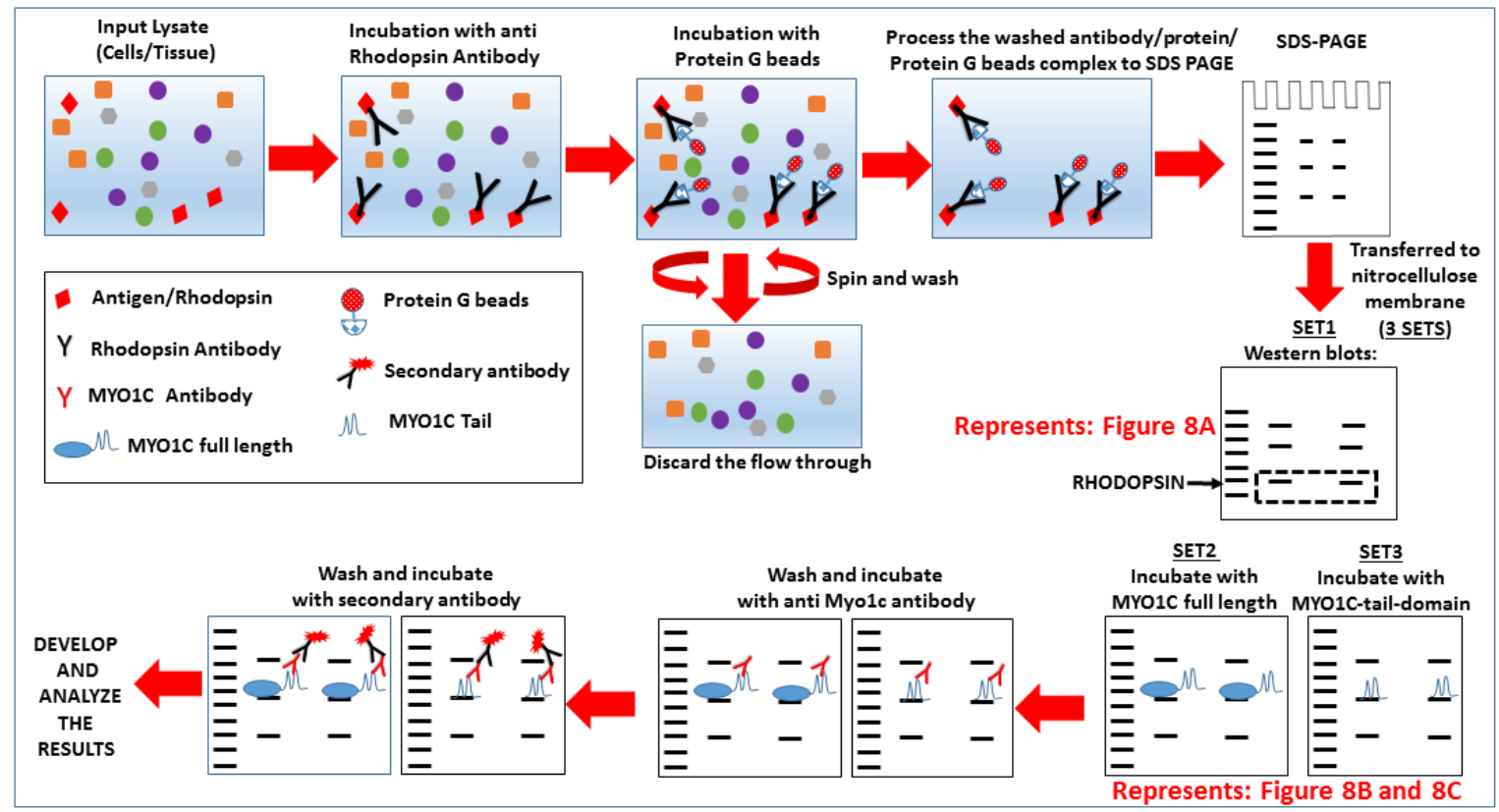

1352

1353

1354

1355

1356

1357

1358

1359

1360

1361

1362

1363

1364

1365 
bioRxiv preprint doi: https://doi.org/10.1101/2020 06.02 129890; this version posted January 15, 2021. The copyright holder for this preprint (which was not certified by peer review) is the author/funder, who has granted bioRxiv a license to display the preprint in perpetuity. It is made available under aCC-BY-NC-ND 4.0 International license.

A

\section{$\stackrel{亠}{\Xi}$}
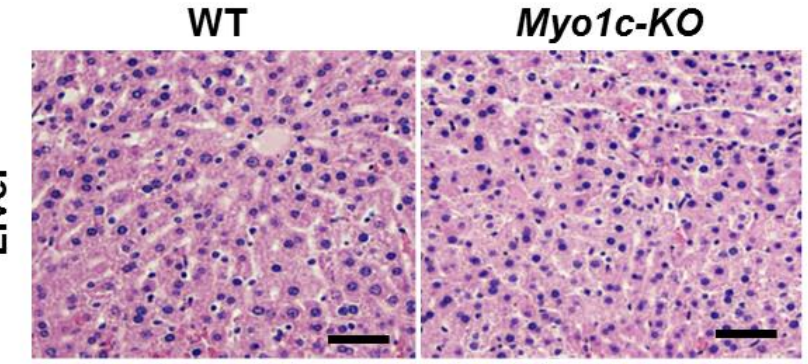

B

亮

C
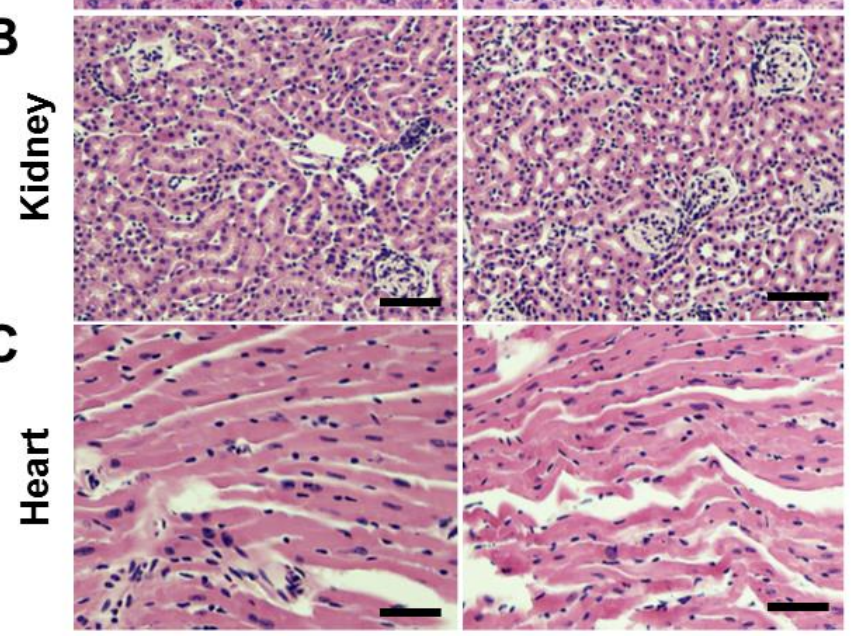

A' $^{\prime}$

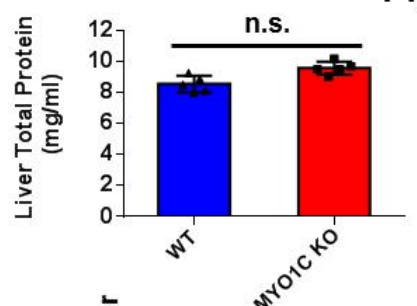

B'
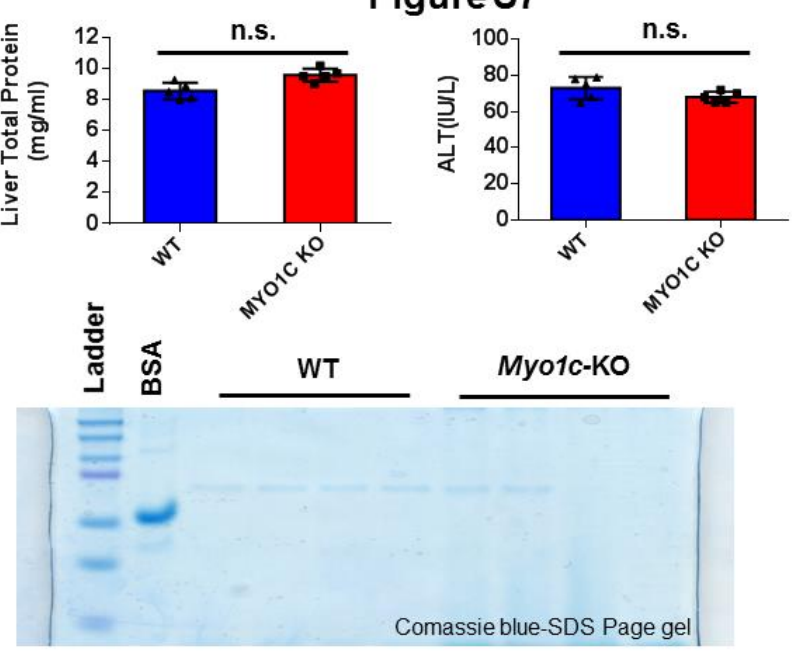

C' B-Mode Long Axis

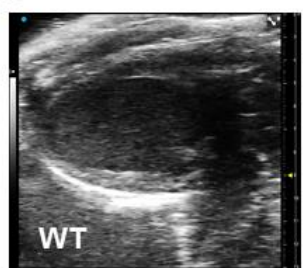

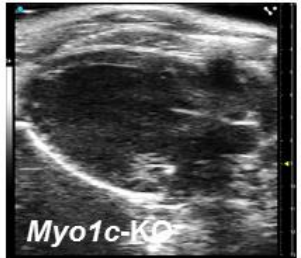

M-Mode Short Axis

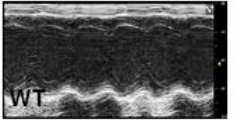

Myote-ko 
A

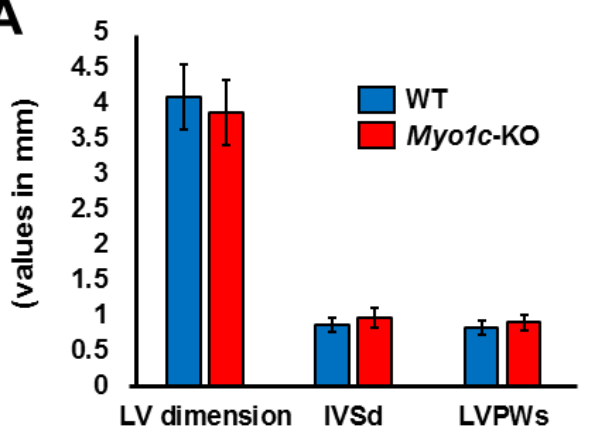

D

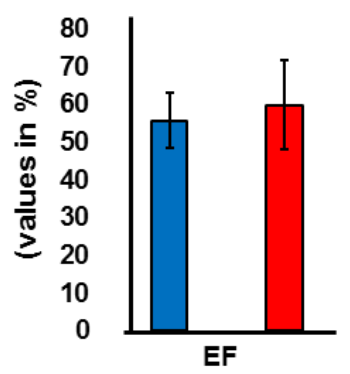

E
B

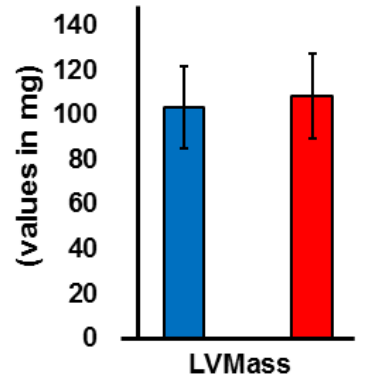

Figure S8

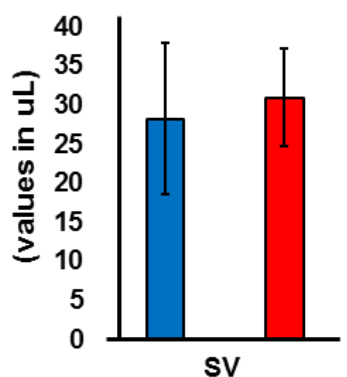

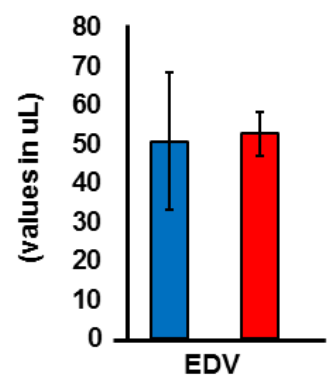

1378

1379

1380

1381

1382

1383

1384

1385

1386

1387
F

\begin{tabular}{|c|c|c|}
\hline Body weight (g) & $27.9 \pm 3.1$ & $25.6 \pm 0.7$ \\
\hline LV dimension at end-diastole $(\mathrm{mm})$ & $4.07 \pm 0.23$ & $3.85 \pm 0.23$ \\
\hline $\begin{array}{l}\text { Septal wall thickness at end-diastole } \\
(\mathrm{mm})\end{array}$ & $0.85 \pm 0.07$ & $0.96 \pm 0.05$ \\
\hline $\begin{array}{l}\text { Posterior wall thickness at end-diastole } \\
(\mathrm{mm})\end{array}$ & $0.83 \pm 0.06$ & $0.89 \pm 0.05$ \\
\hline LV end-diastolic volume $(\mu \mathrm{L})$ & $50.7 \pm 2.8$ & $52.8 \pm 8.8$ \\
\hline Stroke volume $(\mu \mathrm{L})$ & $28.2 \pm 3.1$ & $31.0 \pm 4.8$ \\
\hline LV ejection fraction (\%) & $55.7 \pm 5.9$ & $59.8 \pm 3.6$ \\
\hline LV mass (mg) & $103.3 \pm 9.5$ & $108.1 \pm 9.1$ \\
\hline LV mass to body weight ratio $(\mathrm{mg} / \mathrm{g})$ & $3.76 \pm 0.34$ & $4.20 \pm 0.27$ \\
\hline Sample size & 4 & 4 \\
\hline
\end{tabular}


Figure S9

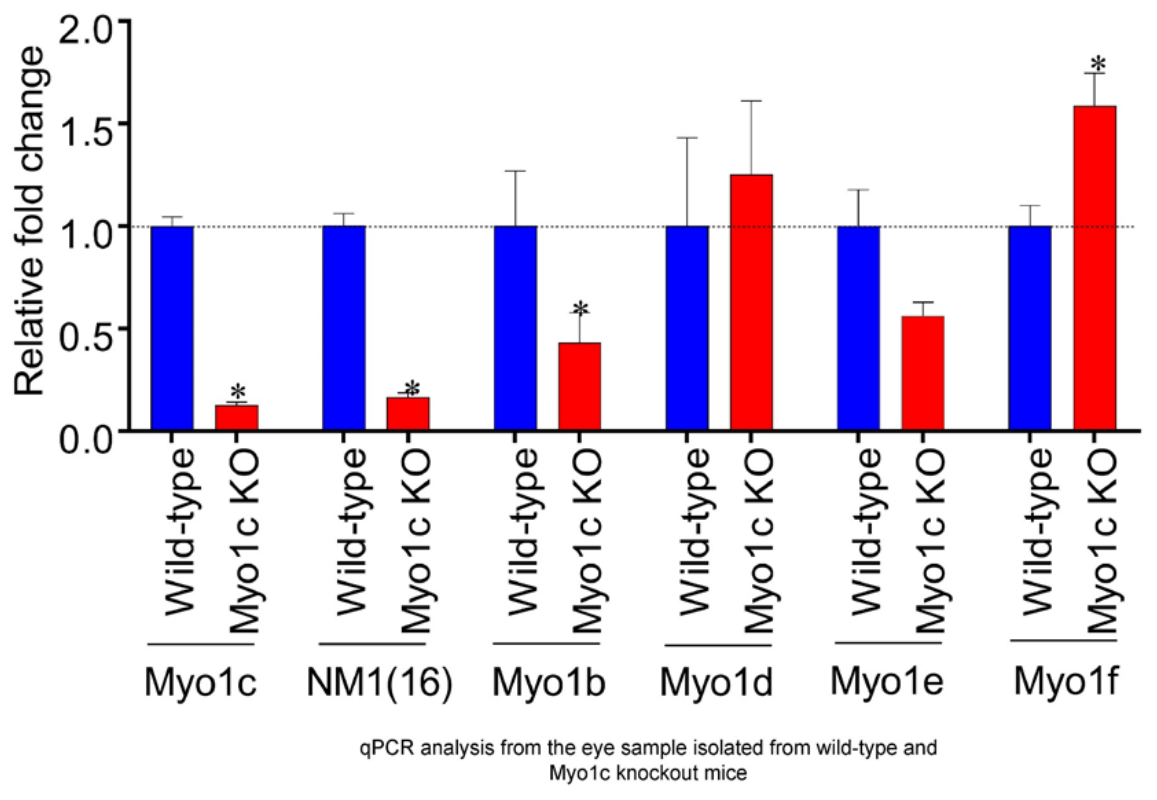

